\title{
Network Formation Games in Cooperative MIMO Interference Systems
}

\author{
Tong Zhou, Yan Chen, Member, IEEE, and K. J. Ray Liu, Fellow, IEEE
}

\begin{abstract}
This paper considers the cooperative optimization of mutual information in the MIMO Gaussian interference channel in a fully distributed manner via game theory. Null shaping constraints are enforced in the design of transmit covariance matrices to enable interference mitigation among links. The transmit covariance matrices leading to the Nash Equilibrium (NE) are derived, and the existence and uniqueness of the $\mathrm{NE}$ is analyzed. The formation of the cooperative sets, that represent the cooperation relationship among links, is considered as coalition games and network formation games. We prove that the proposed coalition formation (CF) and coalition graph formation (CGF) algorithms are Nash-stable, and the proposed network formation (NF) algorithm converges to a Nash Equilibrium. Simulation results show that the proposed CF and CGF algorithms have significant advantages when the antennas at the transmitters is large, and the proposed NF algorithm enhances the sum rate of the system apparently even at low signal-to-noise ratio region and/or with small number of transmit antennas.
\end{abstract}

Index Terms-Game theory, MIMO Interference channel, cooperative network, rate maximization.

\section{INTRODUCTION}

$\mathbf{T}$ HE multiple-input multiple-out (MIMO) interference channel [1] is a mathematical model applicable to many communication systems where multiple links share the same communication medium. Two typical examples are the MIMO cellular systems, where a user located near the cell edge suffers the co-channel interference from the adjacent cell, and the MIMO ad hoc network, where a transceiver pair suffers the interference from other transceiver pairs using the same frequency band. In this model, how to suppress/cancel the cross link interference is crucial, since it greatly impacts the transmission rate. Different techniques have been proposed to counter the inter-cell interference in cellular system including randomization [2], cancellation/rejection [3], coordinated multipoint transmission (CoMP) [4] [5] and inter-cell interference coordination/avoidance (ICIC) in frequency domain [6] and power domain [7]. The work of interference management in the MIMO interference channels includes design of power levels [8][9], precoding matrix [10-16], subcarrier assignment [17], and scheduling [18][19].

In this paper, we focus on the distributed precoding matrix design in the MIMO interference systems. One possible way

Manuscript received April 9, 2013; revised August 29 and October 18, 2013; accepted October 18, 2013. The associate editor coordinating the review of this paper and approving it for publication was D. Niyato.

The authors are with the Department of Electrical and Computer Engineering, University of Maryland, College Park, MD 20742 USA (e-mail: zhoutong2007@gmail.com, \{yan, kjrliu\}@umd.edu). T. Zhou is also with the Key Laboratory of Universal Wireless Communications (Ministry of Education), Beijing University of Posts and Telecommunications, Beijing 100876, China.

Digital Object Identifier 10.1109/TWC.2013.113013.131602 to deal with this problem is to employ interference alignment (IA) [20-22], where the transmitters coordinate so that interfering signals are aligned and seem as originating from a single source. However, IA-based approach requires global channel state information (CSI), which is hard to acquire in practice. Another avenue to deal with the problem is the gametheoretic approach [10-17]. Scutari et al [10][11] formulated the problem as a noncooperative game, designed an iterative waterfilling (IWF) algorithm that was suitable for arbitrary channel matrix to maximize the mutual information, and analyzed the existence and uniqueness of the Nash Equilibrium (NE). This IWF algorithm requires no cross-link CSI and is easy to implement, but the efficiency of the NE is restricted due to the selfishness of the players and the lack of interference coordination. A simple way to improve the efficiency of NE is to introduce pricing [12] for interference management. Interference price was injected into the sum rate maximization objective function with minimum mean-squared error (MMSE) receivers in [13], while the first-order Taylor approximation of sum rate of other links was regarded as the price in [14], and the multi-user sum rate optimization problem was formulated as a concave game to conduct the analysis of the existence and uniqueness of NE. Interference coordination can also be formulated as cooperative games. A practical suboptimal algorithm for finding the Nash Bargaining (NB) solution in MIMO interference system was designed in [15]. The authors of [16] designed the precoding vectors by combining egostic and altruistic beamforming vectors, and this idea has been shown to achieve Pareto boundary in two-player MISO interference systems [23]. The Pareto boundary for multi-player MIMO interference channels was characterized in [24].

Unlike the above works, we consider a new approach of interference coordination, where null shaping constraints are enforced in the design of transmit covariance matrices to enable interference mitigation among different links. In [25], the null shaping constraints are used in cognitive radio scenario to limit the interference of secondary users to the primary users unrequitedly, without cooperation among themselves. In our work, the null shaping constraints are imposed multilaterally. For each transceiver, there is a pair of outgoing cooperative set and incoming cooperative set, which stands for the links alleviating interference to this link and the links profiting from null shaping constraints enforced on this link, respectively.

In this paper, we first formulate the cooperative multi-link transmission with cross-link interference mitigation problem as a strategy game by fixing the outgoing cooperative set and incoming cooperative set. Then the solution leading to the 
$\mathrm{NE}$ is derived, and the uniqueness and existence of the NE is analyzed. Thirdly, we formulate the formation of cooperative sets as coalition games [26-28] and network formation games [29], provide algorithms for these games, and investigate the stability of the proposed algorithms. Simulation results show that the game with null shaping constraints among links improves the probability of uniqueness of the NE, compared to the noncooperative game. The proposed coalition algorithms and network formation algorithms achieve higher average rate than that of the noncooperative waterfilling algorithm. The network formation algorithm improves the sum rate of the system significantly even at low SNR region and/or with small number of transmit antennas.

Throughout this paper, we use upper boldface letter for matrices and lowercase boldface for vectors. $\mathbf{A}^{\mathrm{H}}$ and $\mathbf{A}^{-1}$ refer to the conjugate transpose and Moore-Penros pseudo inverse of a matrix $\mathbf{A}$. $\operatorname{det}(\mathbf{A}), \operatorname{Tr}(\mathbf{A}),\|\mathbf{A}\|_{F}$ and $\rho(\mathbf{A})$ stand for the determinant, trace, Frobenius norm and spectral radius of $\mathbf{A}$. The rank of a matrix $\mathbf{A}$ is denoted as $\operatorname{rank}(A)$ or $r_{A}$. I represents an identity matrix. $(x)^{+} \triangleq \max (0, x)$.

The rest of this paper is outlined as follows. Section II reviews the noncooperative rate maximization game in MIMO interference systems. In Section III, we present the cooperative multi-link MIMO transmission with transmitter null shaping, derive the solution leading to NE, and investigate the condition of the uniqueness and existence of the NE. In Section IV, we model the problem of cooperative sets formation as coalition games in partition form, coalition graph game and network formation game respectively, and propose distributed algorithms for these formulations. Simulation results are demonstrated and analyzed in Section V. Finally, concluding remarks are given in Section VI.

\section{Noncooperative Rate Maximization Game}

We consider a vector Gaussian interference channel comprising $K$ MIMO links that share the same physical resources. In link $q$, the transmitter and receiver are equipped with $n_{T_{q}}$ and $n_{R_{q}}$ antennas respectively. As shown in Fig.1, when a transmitter sends signal to its receiver, it also causes interference to other receivers. The transmission over link $q$ can be described by the baseband signal model

$$
\mathbf{y}_{q}=\mathbf{H}_{q q} \mathbf{x}_{q}+\sum_{r \neq q} \mathbf{H}_{r q} \mathbf{x}_{r}+\mathbf{n}_{q}
$$

where $\mathbf{x}_{q} \in \mathbb{C}^{n_{T_{q}} \times 1}$ is the transmitted signal vector by source $q, \mathbf{n}_{q} \in \mathbb{C}^{n_{R_{q}} \times 1}$ is a zero-mean circularly symmetric complex Gaussian noise vector with nonsingular covariance matrix $\mathbf{R}_{n_{q}} . \mathbf{H}_{q q} \in \mathbb{C}^{n_{R_{q}} \times n_{T_{q}}}$ is the channel matrix of link $q$, $\mathbf{H}_{r q} \in \mathbb{C}^{n_{R_{q}} \times n_{T_{r}}}$ is the cross-channel matrix between source $r$ and destination $q$. We assume that $\mathbf{H}_{r q} \sim \mathcal{C N}\left(0, \eta_{r q}^{2} \mathbf{I}\right)$ $(r, q \in\{1,2, . ., K\})$ with $\eta_{r q}=\sqrt{\kappa / d_{r q}^{\alpha}}$, where $\kappa$ is the path loss constant, $d_{r q}$ is the distance between transmitter $r$ and receiver $q$, and $\alpha$ is the path loss exponent.

The second term in the right handside of (1), $\sum_{r \neq q} \mathbf{H}_{r q} \mathbf{x}_{r}$, represents the co-channel interference received by $q$ th destination. We assume that the co-channel interference from

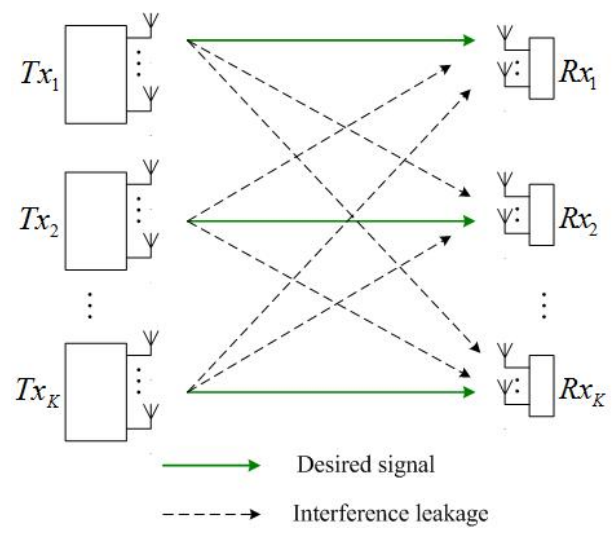

Fig. 1. K-pair MIMO interference channel model.

other links to destination $q$ is unknown and treated as noise. We assume the slow fading channels, i.e., the channels are fixed during a symbol transmission. Moreover, we assume perfect CSI at both transmitter and receiver sides, and each receiver can perfectly measure the covariance matrix of the noise together with co-channel interference generated by other links.

With the above assumption, the maximum information rate on link $q$ can be expressed as [1]:

$$
R_{q}\left(\mathbf{Q}_{q}, \mathbf{Q}_{-q}\right)=\log \operatorname{det}\left(\mathbf{I}+\mathbf{H}_{q q}^{H} \mathbf{R}_{-q}^{-1}\left(\mathbf{Q}_{-q}\right) \mathbf{H}_{q q} \mathbf{Q}_{q}\right)
$$

where $\mathbf{Q}_{q}=\mathbb{E}\left[\mathbf{x}_{q} \mathbf{x}_{q}^{H}\right]$ is the Hermitian positive semi-definite (PSD) transmit covariance matrix of the transmitted vector $\mathbf{x}_{q}$, i.e. $\mathbf{Q}_{q} \succeq 0$, and

$$
\mathbf{R}_{-q}\left(\mathbf{Q}_{-q}\right)=\mathbf{R}_{n_{q}}+\sum_{r \neq q} \mathbf{H}_{r q} \mathbf{Q}_{r} \mathbf{H}_{r q}^{H}
$$

is the interference-plus-noise covariance matrix observed by user $q, \mathbf{Q}_{-q} \triangleq\left(\mathbf{Q}_{r}\right)_{r \neq q}$ is the set of all links' covariance matrices by removing the link $q$. The transmission of each link is power limited, i.e.,

$$
\operatorname{Tr}\left(\mathbf{Q}_{q}\right) \leqslant P_{q}
$$

Given the above setup, the problem can be formulated as a strategic noncooperative game[10][11][30]:

$$
\begin{array}{ccc}
(\mathcal{G}): & \max _{\mathbf{Q}_{q}} & R_{q}\left(\mathbf{Q}_{q}, \mathbf{Q}_{-q}\right) \\
\text { s.t. } & \mathbf{Q}_{q} \in \mathcal{Q}_{q}
\end{array} \quad \forall q \in \Omega
$$

where $\Omega \triangleq\{1, . ., K\}$ is the set of players (i.e., the links), $R_{q}\left(\mathbf{Q}_{q}, \mathbf{Q}_{-q}\right)$ is the payoff function of play $q$ defined in (2), and $\mathcal{Q}_{q}$ is the set of valid strategies (the covariance matrices) of player $q$, defined as

$$
\mathcal{Q}_{q} \triangleq\left\{\mathbf{Q} \in \mathbb{C}^{n_{T_{q}} \times n_{T_{q}}}: \quad \mathbf{Q} \succeq \mathbf{0}, \quad \operatorname{Tr}(\mathbf{Q}) \leqslant P_{q}\right\} .
$$

In the noncooperative game $\mathcal{G}$, each player competes with each other selfishly by choosing his strategy, the transmit covariance matrix $\mathbf{Q}_{q}$, to maximize his own information rate $R_{q}\left(\mathbf{Q}_{q}, \mathbf{Q}_{-q}\right)$ defined in (2), subject to the average transmit power constraint in (4). A Nash Equilibrium is reached when each user, given the strategy profiles of others, does not get any rate increase by unilaterally changing his own strategy 
[12]. The transmit covariance matrix leading to the NE can be found via IWF as [10][11]:

$$
\mathbf{Q}_{q}^{\star}=\mathbf{U}_{q}\left(\mu_{q} \mathbf{I}-\mathbf{D}_{q}^{-1}\right)^{+} \mathbf{U}_{q}^{H}
$$

where $\mathbf{U}_{q} \mathbf{D}_{q} \mathbf{U}_{q}^{H}=\mathbf{H}_{q q}^{H} \mathbf{R}_{-q}^{-1}\left(\mathbf{Q}_{-q}^{\star}\right) \mathbf{H}_{q q}$ is the eigenvalue decomposition (EVD) of $\mathbf{H}_{q q}^{H} \mathbf{R}_{-q}^{-1}\left(\mathbf{Q}_{-q}^{\star}\right) \mathbf{H}_{q q}, \mathbf{U}_{q}$ is the unitary matrix of eigenvectors, $\mathbf{D}_{q}$ is a diagonal matrix of eigenvalues, and $\mu_{q}$ denotes the water level, which is optimized to meet the power constraint, i.e., $\operatorname{Tr}\left(\mathbf{Q}_{q}^{\star}\right)=P_{q}$.

\section{CoOperative Multi-Link MiMO Transmission WITH TRANSMITTER NUll SHAPING}

The strength of desired signal and noise plus cross-link interference are the two main factors affecting the transmission rate. In the noncooperative rate maximization game $\mathcal{G}$, players choose their strategies by maximizing the useful signal power, without considering the cross-link interference, due to which the transmission rate is impaired. To further improve the performance in MIMO interference channel system, we propose a new rate maximization game in this paper, where null shaping constraints are enforced in the design of transmit covariance matrices to enable interference mitigation among links.

\section{A. Problem Formulation}

Let $\mathcal{C}_{q}$ be the incoming cooperative set of links that eliminate co-channel interference to link $q$, and $\mathcal{N}_{q}$ be the outgoing cooperative set of links that profit from null shaping constraints imposed on link $q$. Assume that link $q$ expects to achieve $n_{l_{q}}$ data streams, $n_{l_{q}} \leq \min \left\{n_{T_{q}}, n_{R_{q}}\right\}$, then the outgoing cooperative set $\mathcal{N}_{q}$ should satisfy

$$
\sum_{r \in \mathcal{N}_{q}} n_{R_{r}} \leqslant n_{T_{q}}-n_{l_{q}}
$$

The constraint in (8) restricts the size of outgoing cooperative set $\mathcal{N}_{q}$. The more antennas deployed at the transmitter, the more degree of freedom player $q$ has for others. It should be noted that, the condition in (8) could be made less restrictive when all transmitters in $\mathcal{N}_{q}$ and transmitter $q$ send a single data stream to their receivers [31]. Then the condition in (8) becomes $\left|\mathcal{N}_{q}\right| \leq n_{T_{q}}-1$. However, this zero forcing transmission would require rank constraints on the transmit covariance matrices [32], which is out of the scope of this paper and thus is not considered here.

Given the cooperative sets $\mathcal{C}_{q}$ and $\mathcal{N}_{q}$ for each player, the maximum information rate on link $q$ can be expressed as

$$
\begin{aligned}
& R_{q}^{c}\left(\mathbf{Q}_{q}\left(\mathcal{N}_{q}\right), \mathbf{Q}_{-\mathcal{C}_{q}}\right)= \\
& \quad \log \operatorname{det}\left(\mathbf{I}+\mathbf{H}_{q q}^{H} \mathbf{R}_{-\mathcal{C}_{q}}^{-1}\left(\mathbf{Q}_{-\mathcal{C}_{q}}\right) \mathbf{H}_{q q} \mathbf{Q}_{q}\left(\mathcal{N}_{q}\right)\right),
\end{aligned}
$$

where

$$
\mathbf{R}_{-\mathcal{C}_{q}}\left(\mathbf{Q}_{-\mathcal{C}_{q}}\right)=\mathbf{R}_{n_{q}}+\sum_{r \notin \mathcal{C}_{q}} \mathbf{H}_{r q} \mathbf{Q}_{r} \mathbf{H}_{r q}^{H}
$$

is the interference-plus-noise covariance matrix observed by user $q, \mathbf{Q}_{-\mathcal{C}_{q}} \triangleq\left(\mathbf{Q}_{r}\right)_{r \notin \mathcal{C}_{q}}$ is the set of covariance matrices of links that is not in $\mathcal{C}_{q}$. Compared to (2), the source of interferers diminishes with cost of sacrificing spatial degrees of freedom to help others.
The valid strategies set of player $q$ through cooperation is defined as

$$
\begin{aligned}
\mathcal{Q}_{q}^{c} \triangleq\left\{\mathbf{Q} \in \mathbb{C}^{n_{T_{q}} \times n_{T_{q}}}:\right. & \mathbf{Q} \succeq \mathbf{0}, \operatorname{Tr}(\mathbf{Q}) \leqslant P_{q}, \\
& \left.\mathbf{H}_{q r} \mathbf{Q} \mathbf{H}_{q r}^{H}=\mathbf{0}, \forall r \in \mathcal{N}_{q}\right\} .
\end{aligned}
$$

Compared to the valid strategies set of noncooperative game in (6), additional null constraints corresponding to set $\mathcal{N}_{q}$ are enforced in (11).

Given the rate function in (9) and the constraints in (11), the cooperative transmission problem can be formulated as a strategic game [30]:

$$
\begin{array}{ccl}
\left(\mathcal{G}^{c}\right): & \max _{\mathbf{Q}_{q}} & R_{q}^{c}\left(\mathbf{Q}_{q}\left(\mathcal{N}_{q}\right), \mathbf{Q}_{-\mathcal{C}_{q}}\right) \\
\text { s.t. } & \mathbf{Q}_{q} \in \mathcal{Q}_{q}^{c}
\end{array} \forall q \in \Omega
$$

In game $\mathcal{G}^{c}$, the player chooses the transmit covariance matrix $\mathbf{Q}_{q}$ by maximizing his own information rate $R_{q}^{c}\left(\mathbf{Q}_{q}\left(\mathcal{N}_{q}\right), \mathbf{Q}_{-\mathcal{C}_{q}}\right)$ with the null shaping constraints. Given the cooperative sets, $\mathcal{G}^{c}$ is regarded as a noncooperative game by definition. However, $\mathcal{G}^{c}$ is formulated to solve the cooperative multi-link transmission problem. Hence, in our paper, it is referred to as cooperative transmission game.

\section{B. $N E$ of $\mathcal{G}^{c}$}

To investigate the Nash equilibria of the proposed cooperative transmission game $\mathcal{G}^{c}$, we first introduce some notations. Denote $\mathbf{H}_{q \mathcal{N}_{q}}=\left[\mathbf{H}_{q l_{1}}^{T}, . ., \mathbf{H}_{q l_{\left|\mathcal{N}_{q}\right|}}^{T}\right]^{T}, l_{i} \in \mathcal{N}_{q}$ as the aggregated channel matrix of links in set $\mathcal{N}_{q}$. Let $\Pi_{\mathbf{H}_{q \mathcal{N}_{q}}}^{\perp}=\mathbf{I}-$ $\mathbf{H}_{q \mathcal{N}_{q}}^{H}\left(\mathbf{H}_{q \mathcal{N}_{q}} \mathbf{H}_{q \mathcal{N}_{q}}^{H}\right)^{-1} \mathbf{H}_{q \mathcal{N}_{q}}$ be the the orthogonal projection onto the null space of matrix $\mathbf{H}_{q \mathcal{N}_{q}}$. Since matrix $\Pi_{\mathbf{H}_{q \mathcal{N}_{q}}}^{\perp}$ is Hermitian and Idempotent, we have the following eigenvalue decomposition,

$$
\mathbf{U}_{q}^{\perp} \mathbf{U}_{q}^{\perp H}=\Pi_{\mathbf{H}_{q \mathcal{N}_{q}}}^{\perp} .
$$

where $\mathbf{U}_{q}^{\perp} \in \mathbb{C}^{n_{T_{q}} \times r_{\mathbf{U}_{q}}}$ is the semi-unitary matrix orthogonal to $\mathbf{H}_{q \mathcal{N}_{q}}$, with $r_{\mathbf{U}_{q}^{\perp}} \triangleq \operatorname{rank}\left(\mathbf{U}_{q}^{\perp}\right)=n_{T_{q}}-\operatorname{rank}\left(\mathbf{H}_{q \mathcal{N}_{q}}\right)$. Denote $\tilde{\mathbf{H}}_{q r}=\mathbf{H}_{q r} \mathbf{U}_{q}^{\perp}$ as the modified channel from transmitter $q$ to receiver $r$. The Nash equilibria of the proposed game $\mathcal{G}^{c}$ is shown in the following theorem.

Theorem 1: All the Nash equilibria of the cooperative transmission game $\mathcal{G}^{c}$ are the solutions to the following fixedpoint equations:

$$
\mathbf{Q}_{q}^{c \star}=\mathbf{U}_{q}^{\perp} \tilde{\mathbf{U}}_{q}\left(\tilde{\mu}_{q} \mathbf{I}-\tilde{\mathbf{D}}_{q}^{-1}\right)^{+} \tilde{\mathbf{U}}_{q}^{H} \mathbf{U}_{q}^{\perp H}, \forall q \in \Omega
$$

where $\tilde{\mathbf{U}}_{q} \tilde{\mathbf{D}}_{q} \tilde{\mathbf{U}}_{q}^{H}=\tilde{\mathbf{H}}_{q q}^{H} \mathbf{R}_{-\mathcal{C}_{q}}^{-1}\left(\mathbf{Q}_{-\mathcal{C}_{q}}^{c \star}\right) \tilde{\mathbf{H}}_{q q}$ is the eigenvalue decomposition. $\tilde{\mathbf{U}}_{q}$ is the unitary matrix of eigenvectors, $\tilde{\mathbf{D}}_{q}$ is a diagonal matrix of eigenvalues, and $\tilde{\mu}_{q}$ denotes the water level, which is optimized to meet the power constraint, i.e., $\operatorname{Tr}\left(\mathbf{Q}_{q}^{c \star}\right)=P_{q}$.

Proof: The proof of theorem 1 is similar to [25, Th.1], here, we outline the proof for readability. For a player $q$, he does not cause interference to the links in his outgoing cooperative set $\mathcal{N}_{q}$, which means that $\mathbf{Q}_{q}$ lies in the null space of $\mathbf{H}_{q \mathcal{N}_{q}}$. Thus the best response strategy of player $q$ in game $\mathcal{G}^{c}$ can be expressed as

$$
\mathbf{Q}_{q}^{c}=\mathbf{U}_{q}^{\perp} \tilde{\mathbf{Q}}_{q}^{c} \mathbf{U}_{q}^{\perp H} .
$$


Since $\mathbf{U}_{q}^{\perp H} \mathbf{U}_{q}^{\perp}=\mathbf{I}$, we can get $\operatorname{Tr}\left(\mathbf{Q}_{q}^{c}\right)=$ $\operatorname{Tr}\left(\mathbf{U}_{q}^{\perp} \tilde{\mathbf{Q}}_{q}^{c} \mathbf{U}_{q}^{\perp H}\right)=\operatorname{Tr}\left(\tilde{\mathbf{Q}}_{q}^{c}\right)$, and the feasible region of $\tilde{\mathbf{Q}}_{q}^{c}$ is

$$
\tilde{\mathcal{Q}}_{q}^{c}=\left\{\mathbf{Q} \in \mathbb{C}^{r} \mathbf{U}_{q}^{\perp} \times r_{\mathbf{U}^{\perp}}: \mathbf{Q} \succeq \mathbf{0}, \operatorname{Tr}(\mathbf{Q}) \leqslant P_{q}\right\} .
$$

And $\tilde{\mathbf{Q}}_{q}^{c}$ is the solution of the following optimization problem.

$$
\begin{array}{ll}
\max _{\tilde{\mathbf{Q}}_{q} \succeq \mathbf{0}} & \log \operatorname{det}\left(\mathbf{I}+\mathbf{H}_{q q}^{H} \tilde{\mathbf{R}}_{-\mathcal{C}_{q}}^{-1}\left(\tilde{\mathbf{Q}}_{-\mathcal{C}_{q}}\right) \mathbf{H}_{q q} \mathbf{U}_{q}^{\perp} \tilde{\mathbf{Q}}_{q} \mathbf{U}_{q}^{\perp H}\right) \\
\text { s.t. } & \tilde{\mathbf{Q}}_{q} \in \tilde{\mathcal{Q}}_{q}^{c},
\end{array}
$$

where,

$$
\begin{aligned}
\tilde{\mathbf{R}}_{-\mathcal{C}_{q}}\left(\tilde{\mathbf{Q}}_{-\mathcal{C}_{q}}\right) & =\mathbf{R}_{-\mathcal{C}_{q}}\left(\mathbf{Q}_{-\mathcal{C}_{q}}\right) \\
& =\mathbf{R}_{n_{q}}+\sum_{r \notin \mathcal{C}_{q}} \mathbf{H}_{r q} \mathbf{U}_{r}^{\perp} \tilde{\mathbf{Q}}_{r} \mathbf{U}_{r}^{\perp H} \mathbf{H}_{r q}^{H} .
\end{aligned}
$$

The solution of (17) is [10][11]:

$$
\tilde{\mathbf{Q}}_{q}^{c}=\tilde{\mathbf{U}}_{q}\left(\tilde{\mu}_{q} \mathbf{I}-\tilde{\mathbf{D}}_{q}^{-1}\right)^{+} \tilde{\mathbf{U}}_{q}^{H}, \forall q \in \Omega
$$

where $\tilde{\mathbf{U}}_{q} \tilde{\mathbf{D}}_{q} \tilde{\mathbf{U}}_{q}^{H}=\mathbf{U}_{q}^{\perp H} \mathbf{H}_{q q}^{H} \tilde{\mathbf{R}}_{-\mathcal{C}_{q}}^{-1}\left(\tilde{\mathbf{Q}}_{-\mathcal{C}_{q}}^{c}\right) \mathbf{H}_{q q} \mathbf{U}_{q}^{\perp}$ is the eigenvalue decomposition. $\tilde{\mathbf{U}}_{q}$ is the unitary matrix of eigenvectors, $\tilde{\mathbf{D}}_{q}$ is a diagonal matrix of eigenvalues, and $\tilde{\mu_{q}}$ denotes the water level, which is optimized to meet the power constraint.

Substituting (19) into (15), all the Nash equilibria of game $\mathcal{G}^{c}$ are the solutions to the fixed-point equations in (14).

\section{Conditions for the Existence and Uniqueness of the NE}

In this subsection, we analyze the existence and uniqueness of the NE of the cooperative transmission game $\mathcal{G}^{c}$.

Theorem 2 (Existence): In the game $\mathcal{G}^{c}$, there always exists a NE, for any given cooperative sets, channel matrices and power constraints.

Proof: In game $\mathcal{G}^{c}$, the payoff functions is quasi-concave and the valid strategy sets are convex compact, thus there always exists a NE for any modified channel matrices and power constraints [11].

To analyze the condition for the uniqueness of the NE of the game $\mathcal{G}^{c}$, we introduce a nonnegative matrix $\mathbf{S}^{c} \in \mathbb{C}^{K \times K}$ as follows

$$
\left[\mathbf{S}^{c}\right]_{q r} \triangleq \begin{cases}\rho\left(\tilde{\mathbf{H}}_{r q}^{H} \tilde{\mathbf{H}}_{q q}^{-H} \tilde{\mathbf{H}}_{q q}^{-1} \tilde{\mathbf{H}}_{r q}\right), & \text { if } r \neq q \\ 0, & \text { otherwise }\end{cases}
$$

where $\tilde{\mathbf{H}}_{r q}=\mathbf{H}_{r q} \mathbf{U}_{r}^{\perp}$ is the modified channel from transmitter $r$ to receiver $q$. With the matrix $\mathbf{S}^{c}$, we can obtain sufficient conditions for the uniqueness of the NE of the proposed cooperative transmission game $\mathcal{G}^{c}$ as shown in the following theorem.

Theorem 3 (Uniqueness): The NE of the cooperative transmission game $\mathcal{G}^{c}$ is unique if

$$
\rho\left(\mathbf{S}^{c}\right)<1
$$

The proof of Theorem 3 is similar to [11, Th.6][25,Th.1].

\section{Cooperative Sets Formation}

In the previous section, we discuss the formulation and properties of the proposed cooperative transmission game assume that the cooperative sets are given. However, how to acquire the cooperative set is very important. In this section, we formulate the cooperative set formation as coalition games and network formation games, and design corresponding distributed algorithms.

\section{A. Cooperative Sets Formation using Coalition Formation Game}

Given a partition $\Pi$ of $\Omega$ and a coalition $S \in \Pi$, we denote $v_{q}(S, \Pi)$ as the payoff that link $q \in S$ can receive when acting in coalition $S$ with the partition $\Pi$. Consider the transmission rate as the payoff of each player, the coalition value set, i.e., the mapping $V$, can be defined as follows:

$$
\begin{aligned}
V(S, \Pi)= & \left\{\mathbf{v}(S, \Pi) \in \mathbb{R}^{|S|} \mid v_{q}(S, \Pi)=R_{q}^{c}\left(\mathbf{Q}_{q}(S), \mathbf{Q}_{-S}\right),\right. \\
& \forall q \in S\} .
\end{aligned}
$$

Using (22), the cooperative set formation can be modeled as a $(\Omega, V)$ coalition game in partition form with nontransferable utility[26]. With such a formulation, the two cooperative sets, $\mathcal{C}_{q}$ and $\mathcal{N}_{q}$, are the same, i.e., $\mathcal{C}_{q}=\mathcal{N}_{q}=S \backslash\{q\}$.

In order to build a coalition formation algorithm for the cooperative sets formation, we define the following coalition switch rule.

Definition 1 (Coalition Switch Rule): Given a partition $\Pi=\left\{S_{1}, . ., S_{M}\right\}$ of the set of links $\Omega$, a link $q$ decides to leave its current coalition $S_{m}$ and join another coalition $S_{k} \in$ $\Pi \cup\{\varnothing\}, S_{k} \neq S_{m}$, leading to a new partition $\Pi^{\prime}=\{\Pi \backslash$ $\left.\left\{S_{m}, S_{k}\right\}\right\} \cup\left\{S_{m} \backslash\{q\}, S_{k} \cup\{q\}\right\}$, if and only if,

$$
\left\{\begin{array}{l}
v_{q}\left(S_{k} \cup\{q\}, \Pi^{\prime}\right)>v_{q}\left(S_{m}, \Pi\right), q \in S_{m} \\
v_{r}\left(S_{k} \cup\{q\}, \Pi^{\prime}\right) \geqslant v_{r}\left(S_{k}, \Pi\right), \forall r \in S_{k} \\
S_{k} \cup\{q\} \notin h(q), \text { if } S_{k} \neq \varnothing
\end{array}\right.
$$

The first condition in (23) indicates that the payoff of player $q$ can be strictly improved after joining another coalition while the second one guarantees that the payoff of players in the new coalition will not decrease. The third constraint is used to avoid repetitive partitions, where $h(q)$ is the history set of the coalitions that player $q$ belongs to. The three conditions are also defined as preference relation [27][33], and can be denoted as $\left(S_{k} \cup\{q\}, \Pi^{\prime}\right) \succ_{q}\left(S_{m}, \Pi\right)$ for simplicity.

Based on the coalition switch rule, we present a coalition formation (CF) algorithm which consists of three phases: neighbor discovery, coalition formation, and MIMO transmission with transmitter null shaping.

In Phase 1, each player discovers its strongest interferers. Denote $p_{r q}$ as the average signal/interference power received by player $q$ from transmitter $r$. For player $q$, if $p_{r q} / p_{q q}>\alpha$, he will add this link in his possible incoming cooperative set $\tilde{\mathcal{C}}_{q}$.

In Phase 2, players investigate the possibility of coalition switch by pairwise negotiation with the neighbors detected in Phase 1. We assume that players perform coalition switch distributedly and sequentially with random orders. The effect of different orders on the performance will be discussed 
in section V. For simplicity, a player only investigates the one including his strongest interferer instead of all possible coalitions. A procedure of coalition formation is described as follow.

For a player $q$, we denote $S^{q}$ as his coalition member set, $S^{q}=S_{m} \backslash\{q\}, q \in S_{m}$.

1. Player $q$ checks whether his possible incoming cooperative set $\tilde{\mathcal{C}}_{q}$ and coalition member set $S^{q}$ are the same. If not, he will find the strongest interferer in the set $\tilde{\mathcal{C}}_{q} \backslash S^{q}$, denoted as $\pi_{q}$. Then an INQUIRE request is sent to this interferer.

2. After player $\pi_{q}$ receives the request, he will send his coalition information $\left(S_{k}=\pi_{q} \cup S^{\pi_{q}}\right)$ to player $q$, and forward the INQUIRE request to other members in $S_{k}$.

3. Player $q$ gets the information of target coalition, and checks the third condition in (23), i.e., $S_{k} \cup\{q\} \notin h(q)$. If it is satisfied, he will estimate his payoff in (22) assuming that a new partition $\Pi^{\prime}=\left\{\Pi \backslash\left\{S_{m}, S_{k}\right\}\right\} \cup\left\{S_{m} \backslash\{q\}, S_{k} \cup\{q\}\right\}$ is constructed. This indicates that set $S^{q}$ stops interference cancelling to link $q$, and players in $S_{k}$ start to perform null shaping to him. To do this, player $q$ needs to acquire the channel matrices $\left\{\mathbf{H}_{q r}\right\}_{r \in S_{k}}$ for generating $\mathbf{U}_{q}^{\perp}$, and estimates the interference-plus-noise covariance matrix $\mathbf{R}_{-S_{k} \cup\{q\}}\left(\mathbf{Q}_{-S_{k} \cup\{q\}}\right)$. If $v_{q}\left(S_{k} \cup\{q\}, \Pi^{\prime}\right)>v_{q}\left(S_{m}, \Pi\right)$, player $q$ will send members in $S_{k}$ a JOIN request. In the meantime, each player $r \in S_{k}$ calculates his payoff in (22) assuming that a new coalition $S_{k} \cup\{q\}$ is formed. If $v_{r}\left(S_{k} \cup\{q\}, \Pi^{\prime}\right) \geqslant v_{r}\left(S_{k}, \Pi\right)$, he will send player $q$ and other members in $S_{k}$ an AGREE message.

4. if player $q$ receives all the AGREE messages from members in the target coalition, he will update his coalition member set $S^{q}=S_{k}$. And If $\left|S_{m}\right|>1$, he will send a DEVIATE request to players in $S^{q}$, and update his history $h(q)=h(q) \cup\left\{S_{m}\right\}$. Meantime, if player $r \in S_{k}$ receives the JOIN request from player $q$ and all the AGREE message from other players in $S_{k}$, he will update his coalition member set $S^{r}=S^{r} \cup\{q\}$. A new partition $\Pi^{\prime}=\left\{\Pi \backslash\left\{S_{m}, S_{k}\right\}\right\} \cup$ $\left\{S_{m} \backslash\{q\}, S_{k} \cup\{q\}\right\}$ is generated. Denote $S_{q, l}$ as the last history of coalition put into set $h(q)$, Phase 2 ends until $\left|v_{q}\left(S^{q} \cup\{q\}\right)-v_{q}\left(S_{q, l}\right)\right|<\eta, \forall q \in \Omega . \eta$ is the threshold for determining convergence.

In Phase 3, players transmit data cooperatively in MIMO interference channel, imposing null shaping constraints formed in Phase 2 on the transmitters. There may be multiple NEs. In such cases, we do not search all the NEs and find the best one. Instead, we stop Phase 3 once a NE is found for simplicity, due to which the achieved NE may depend on the initial conditions of the algorithm. Denote $\eta_{N}$ as the maximal number of iterations. If no NE is found within $\eta_{N}$ iterations, Phase 3 stops and each player chooses the strategy in the last iteration.

The proposed coalition formation algorithm is guaranteed to converge as shown in the following theorem.

Theorem 4: The proposed coalition formation algorithm always converges with any initial partition.

Proof: Since the total number of the partitions is finite and the third condition in (23) rules out repetitive deviations, the proposed coalition formation algorithm is guaranteed to converges regardless the initial partition.

The stability of the partition generated from the proposed

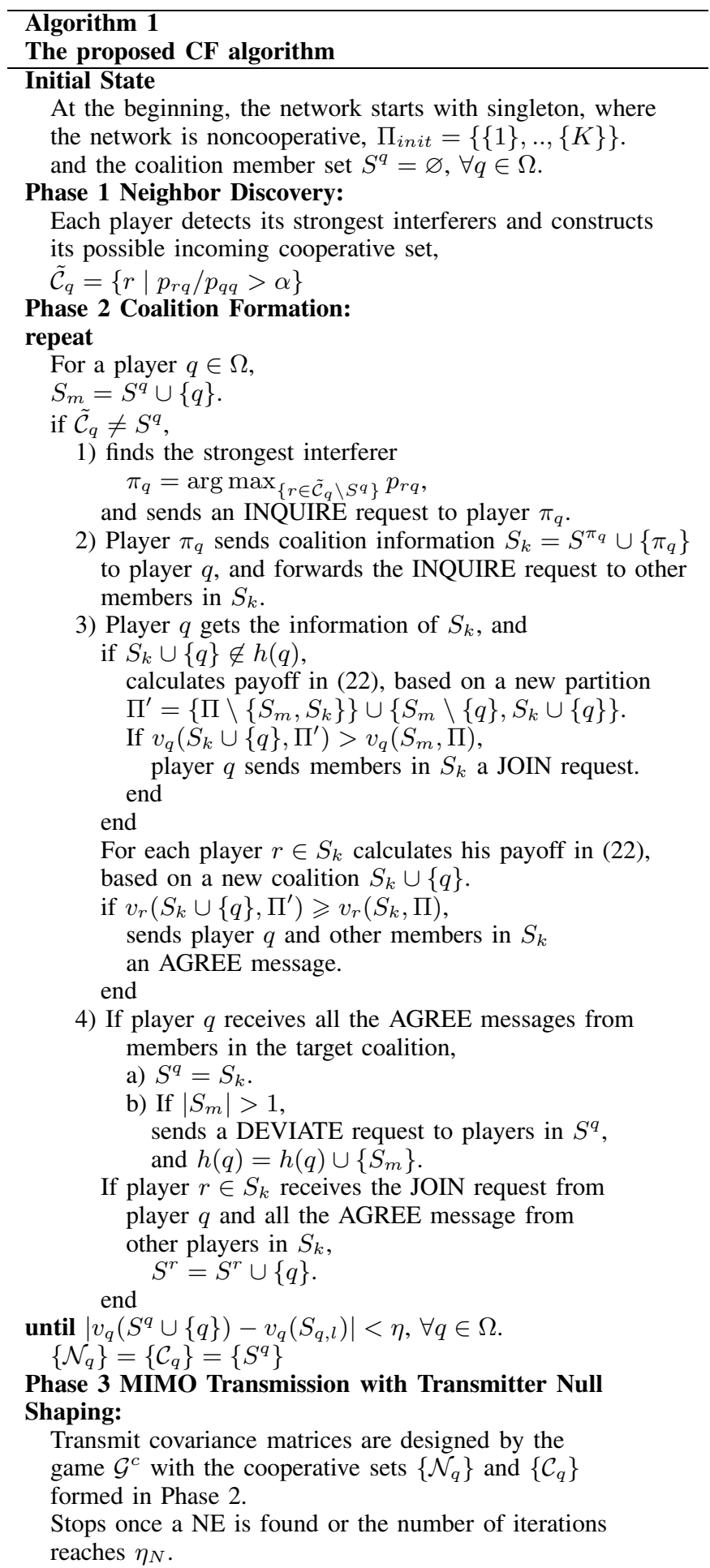

coalition formation algorithm can be evaluated using the concept of Nash-stable [27][33].

Definition 2: A partition $\Pi=\left\{S_{1}, . ., S_{M}\right\}$ is Nash-stable, if $\forall q \in \Omega, q \in S_{m}, S_{m} \in \Pi$, we have $\left(S_{m}, \Pi\right) \succeq_{q}\left(S_{k} \cup\{q\}, \Pi^{\prime}\right)$ for all $S_{k} \in \Pi \cup\{\emptyset\}$ with $\Pi^{\prime}=\Pi \backslash\left\{S_{m}, S_{k}\right\} \cup\left\{S_{m} \backslash\{q\}, S_{k} \cup\right.$ $\{q\}\}$.

From the above definition, we can see that a partition is Nash-stable if no player has the incentive to deviate from its current coalition to join another coalition or to act noncooperatively by forming an individual coalition. Since our coalition formation algorithm is based on the coalition switch 
rule, it is easy to show that the resulting partition is Nashstable.

Proposition 1: The partition derived by the proposed coalition formation algorithm is Nash-stable.

\section{B. Cooperative Sets Formation using Coalition Graph Game}

In the previous subsection, we discuss cooperative sets formation using coalition formation game and propose a coalition formation $(\mathrm{CF})$ algorithm. In the proposed $\mathrm{CF}$ algorithm, we assume that any two players in a coalition help each other, i.e., the cross-link interference between any two players in a coalition is mitigated. The outgoing/incoming cooperative set of a player can be represented by all members of the coalition without any structure among members. In this subsection, we will formulate the cooperative sets formation problem as a coalition graph formation game and propose a coalition graph formation (CGF) algorithm. In the proposed CGF algorithm, whether the cross-link interference between two players in a coalition is canceled or not depends on the connectivity between these two players. The cooperative sets of a player is revealed by the graph structure of members in the coalition.

We first introduce some basic concepts of coalition graph game. A coalition graph $G(S)$ is defined as a pair $(S, A(S))$, where $S$ is a non-empty finite set of vertices, and $A(S)$ is a collection of undirected arcs of the graph. Denote $(q, r)$ as the edge between vertex $q$ and vertex $r$, and the adjacent-vertex set of vertex $q$ in the graph $G(S)$ is denoted as $v t x(G(S), q)=$ $\{r \in S \mid(q, r) \in A(S), q \in S, r \neq q\}$. The set of possible edges between vertex $q$ and the vertices in a coalition $S$ is defined as $\bar{A}(S, q)=\{(q, r) \mid r \in S, q \notin S\}$.

Given a partition $\Pi$ of $\Omega$, a coalition $S \in \Pi$ and a graph $G(S)=(S, A(S))$, we denote $v_{q}(G(S), \Pi)$ as the payoff that link $q \in S$ can receive when acting in coalition graph $G(S)$ with the partition $\Pi$. Consider the transmission rate as the payoff of each player, the coalition value set, i.e., the mapping $V$, can be defined as follows:

$$
V(G(S), \Pi)=\left\{\mathbf{v}(G(S), \Pi) \in \mathbb{R}^{|S|}\right\}
$$

where $\forall q \in S, v_{q}(G(S), \Pi)$ is given by

$$
v_{q}(G(S), \Pi)=R_{q}^{c}\left(\mathbf{Q}_{q}(v t x(G(S), q)), \mathbf{Q}_{-v t x(G(S), q)}\right)
$$

Using (24), the cooperative set formation can be modeled as a $(\Omega, A(\Omega), V)$ coalition graph game [26] in partition form with nontransferable utility. With such a formulation, the two cooperative sets, $\mathcal{C}_{q}$ and $\mathcal{N}_{q}$, are the same, i.e., $\mathcal{C}_{q}=\mathcal{N}_{q}=$ $v t x(G(S), q)$.

In order to present a coalition graph formation algorithm for the cooperative sets formation, we define the following coalition graph switch rule.

Definition 3 (Coalition Graph Switch Rule): Given a partition $\Pi=\left\{S_{1}, . ., S_{M}\right\}$ and the corresponding coalition graph $G(\Pi)=\left\{G\left(S_{1}\right), . ., G\left(S_{M}\right)\right\}$, a link $q$ decides to leave its current coalition $S_{m}$, and join another coalition $S_{k} \in \Pi \cup\{\varnothing\}$, leading to a new partition $\Pi^{\prime}=\{\Pi \backslash$ $\left.\left\{S_{m}, S_{k}\right\}\right\} \cup\left\{S_{m} \backslash\{q\}, S_{k} \cup\{q\}\right\}$ and its corresponding graph
$G\left(\Pi^{\prime}\right)$, if and only if,

$$
\left\{\begin{array}{l}
v_{q}\left(G\left(S_{k}\right) \cup \bar{A}^{\star}\left(S_{k}, q\right), \Pi^{\prime}\right)>v_{q}\left(G\left(S_{m}\right), \Pi\right), q \in S_{m} \\
v_{r}\left(G\left(S_{k}\right) \cup \bar{A}^{\star}\left(S_{k}, q\right), \Pi^{\prime}\right) \geqslant v_{r}\left(G\left(S_{k}\right), \Pi\right), \forall r \in S_{k} \\
G\left(S_{k}\right) \cup \bar{A}^{\star}\left(S_{k}, q\right) \notin h(q), \text { if } S_{k} \neq \varnothing
\end{array}\right.
$$

where,

$$
\left.\bar{A}^{\star}\left(S_{k}, q\right), \Pi^{\prime}\right)=\arg \max _{\bar{a} \subseteq \bar{A}\left(S_{k}, q\right)} v_{q}\left(G\left(S_{k}\right) \cup \bar{a}, \Pi^{\prime}\right) .
$$

The first condition in (26) indicates that the payoff of player $q$ can be strictly improved after removing edges in current coalition and building new edges to another coalition while the second one guarantees the payoff of players in the new coalition will not decrease. The third constraint is used to avoid repetitive partitions. The three conditions in (26) is also called preference relation, and can be denoted as $\left(G\left(S_{k} \cup\right.\right.$ $\left.\{q\}), \Pi^{\prime}\right) \succ_{q}\left(G\left(S_{m}\right), \Pi\right)$ for simplicity.

Based on the coalition graph switch rule, we propose a coalition graph formation (CGF) algorithm composed of three phases: neighbor discovery, coalition graph formation, and MIMO transmission with transmitter null shaping.

In Phase 1, each player discovers its strongest interferers. For player $q$, if $p_{r q} / p_{q q}>\alpha$, he will add this link in his possible incoming cooperative set $\tilde{\mathcal{C}}_{q}$.

In Phase 2, players investigate the possibility of coalition graph switch by pairwise negotiation with the neighbors detected in Phase 1. A procedure of coalition graph formation is described as follow.

For a player $q, S_{m}=S^{q} \cup\{q\}$.

1. He checks whether his possible incoming cooperative set $\tilde{\mathcal{C}}_{q}$ and coalition member set $S^{q}$ are the same. If not, he will find the strongest interferer $\pi_{q}$ in the set $\tilde{\mathcal{C}}_{q} \backslash S^{q}$. Then an INQUIRE request is sent to this interferer.

2. After player $\pi_{q}$ receives the request, he will send his coalition information $\left(S_{k}=\pi_{q} \cup S^{\pi_{q}}\right)$ to player $q$.

3. Player $q$ gets the information of target coalition, and determines the best edge set $\bar{A}^{\star}\left(S_{k}, q\right)$. If $G\left(S_{k}\right) \cup \bar{A}^{\star}\left(S_{k}, q\right) \notin$ $h(q)$, player $q$ will estimate his payoff in (25) assuming that a new coalition graph $G\left(S_{k}\right) \cup \bar{A}^{\star}\left(S_{k}, q\right)$ is constructed. This indicates that set $v t x\left(G\left(S_{m}\right), q\right)$ stops interference cancelling to link $q$, and players in $v \operatorname{tx}\left(\bar{A}^{\star}\left(S_{k}, q\right), q\right)$ start to perform null shaping to him. To do this, player $q$ needs to acquire the channel matrices $\left\{\mathbf{H}_{q r}\right\}_{r \in v t x}\left(\bar{A}^{\star}\left(S_{k}, q\right), q\right)$ for generating $\mathbf{U}_{q}^{\perp}$, and estimates the interference-plus-noise covariance matrix $\mathbf{R}_{-v t x\left(\bar{A}^{\star}\left(S_{k}, q\right), q\right)}\left(\mathbf{Q}_{-v t x\left(\bar{A}^{\star}\left(S_{k}, q\right), q\right)}\right)$. If $v_{q}\left(G\left(S_{k}\right) \cup\right.$ $\left.\bar{A}^{\star}\left(S_{k}, q\right), \Pi^{\prime}\right)>v_{q}\left(G\left(S_{m}\right), \Pi\right)$, a JOIN request is sent to members in $S_{k}$, and a CONNECT request is sent to players in $v t x\left(\bar{A}^{\star}\left(S_{k}, q\right), q\right)$.

4. Each player $r \in S_{k}$ determines the new coalition graph $G\left(S_{k}\right) \cup \bar{A}^{\star}\left(S_{k}, q\right)$ corresponding to the received CONNECT request, and calculates his payoff in (25) based on the new graph. If $v_{r}\left(G\left(S_{k}\right) \cup \bar{A}^{\star}\left(S_{k}, q\right), \Pi^{\prime}\right) \geqslant v_{r}\left(G\left(S_{k}\right), \Pi\right)$, he will send player $q$ and other members in $S_{k}$ an AGREE message.

5. If player $q$ receives all the AGREE messages from members in the target coalition, he will update his coalition member set $S^{q}=S_{k}$, and adjacent-vertex set $v \operatorname{tx}\left(G\left(S_{k} \cup\{q\}\right), q\right)=$ $v \operatorname{tx}\left(\bar{A}^{\star}\left(S_{k}, q\right), q\right)$. And If $\left|S_{m}\right|>1$, he will send a DEVIATE request to players in $S^{q}$, and update his history $h(q)=h(q) \cup$ 
$\left\{G\left(S_{m}\right)\right\}$. Meantime, if player $r \in S_{k}$ receives all the AGREE message from other players in $S_{k}$, he will update his coalition member set $S^{r}=S^{r} \cup\{q\}$, and if the CONNECT request from player $q$ is received in step 4 , he will update his adjacentvertex set $v t x\left(G\left(S_{k} \cup\{q\}\right), r\right)=v t x\left(G\left(S_{k}\right), r\right) \cup\{q\}$. A new partition $\Pi^{\prime}=\left\{\Pi \backslash\left\{S_{m}, S_{k}\right\}\right\} \cup\left\{S_{m} \backslash\{q\}, S_{k} \cup\{q\}\right\}$ and its corresponding graph $G\left(\Pi^{\prime}\right)$ are generated. Denote $G_{q, l}$ as the last history of coalition graph put into set $h(q)$, Phase 2 ends until $\left|v_{q}\left(G\left(S^{q} \cup\{q\}\right)\right)-v_{q}\left(G_{q, l}\right)\right|<\eta, \forall q \in \Omega$.

In Phase 3, players transmit data cooperatively in MIMO interference channel, imposing null shaping constraints formed in Phase 2 on the transmitters. There may be multiple NEs. In such cases, we do not search all the NEs and find the best one. Instead, we stop Phase 3 once a NE is found for simplicity, due to which the achieved NE may depend on the initial conditions of the algorithm. If no NE is found within $\eta_{N}$ iterations, Phase 3 stops and each player chooses the strategy in the last iteration.

The proposed coalition graph formation algorithm is guaranteed to converge as shown in the following theorem:

Theorem 5: The proposed coalition graph formation algorithm always converges with any initial partition.

Proof: Since the total number of coalition graphs is finite and the third condition in (26) rules out repetitive deviations, the proposed coalition graph formation algorithm is guaranteed to converges regardless the initial partition.

The stability of the coalition graph generated from the proposed coalition graph formation algorithm can be evaluated using the expanding concept of Nash-stable.

Definition 4: A coalition graph $G(\Pi)=\left\{G\left(S_{1}\right), . .,\left(S_{M}\right)\right\}$ is Nash-stable, if $\forall q \in \Omega, q \in S_{m}, G\left(S_{m}\right) \in G(\Pi)$, we have $\left(G\left(S_{m}\right), \Pi\right) \succeq_{q}\left(G\left(S_{k}\right) \cup \bar{A}\left(S_{k}, q\right), \Pi^{\prime}\right)$ for all $S_{k} \in \Pi \cup\{\emptyset\}$ with $\Pi^{\prime}=\Pi \backslash\left\{S_{m}, S_{k}\right\} \cup\left\{S_{m} \backslash\{q\}, S_{k} \cup\{q\}\right\}$ and $G\left(\Pi^{\prime}\right)=$ $G(\Pi) \backslash\left\{G\left(S_{m}\right), G\left(S_{k}\right)\right\} \cup\left\{G\left(S_{m}\right) \backslash A\left(S_{m}, q\right), S_{k} \cup \bar{A}\left(S_{k}, q\right)\right\}$.

From the above definition, we can see that a coalition graph is Nash-stable if no player has the incentive to remove edges in its current coalition and build new edges in another coalition or to act non-cooperatively by forming an individual coalition.

Proposition 2: The graph derived by the proposed coalition graph formation algorithm is Nash-stable.

Proof: If the coalition graph $G\left(\Pi_{f}\right)$ resulting from the proposed algorithm is not Nash-stable then $\exists q \in \Omega$ with $q \in$ $S_{m}, G\left(S_{m}\right) \in G\left(\Pi_{f}\right)$, and a coalition graph $G\left(S_{k}\right) \in G\left(\Pi_{f}\right)$ such that $\left(G\left(S_{k}\right) \cup \bar{A}\left(S_{k}, r\right), \Pi^{\prime}\right) \succ_{q}\left(G\left(S_{m}\right), \Pi_{f}\right)$, and player $q$ has the incentive to perform a graph switch operation, and this contradicts with the assumption that $\Pi_{f}$ is the result of the convergence of the proposed algorithm. Hence, any coalition graph achieved by the proposed coalition graph formation algorithm is Nash-stable.

\section{Cooperative Sets Formation using Network Formation Game}

In the above two subsections, we discuss cooperative sets formation using coalition formation game and coalition graph formation game. In these two coalition games, players in one coalition are balanced, i.e., if player $q$ helps player $r, r \neq q$, player $r$ will also help player $q$. But in some cases, helps can be nonreciprocal. In this subsection, we formulate the

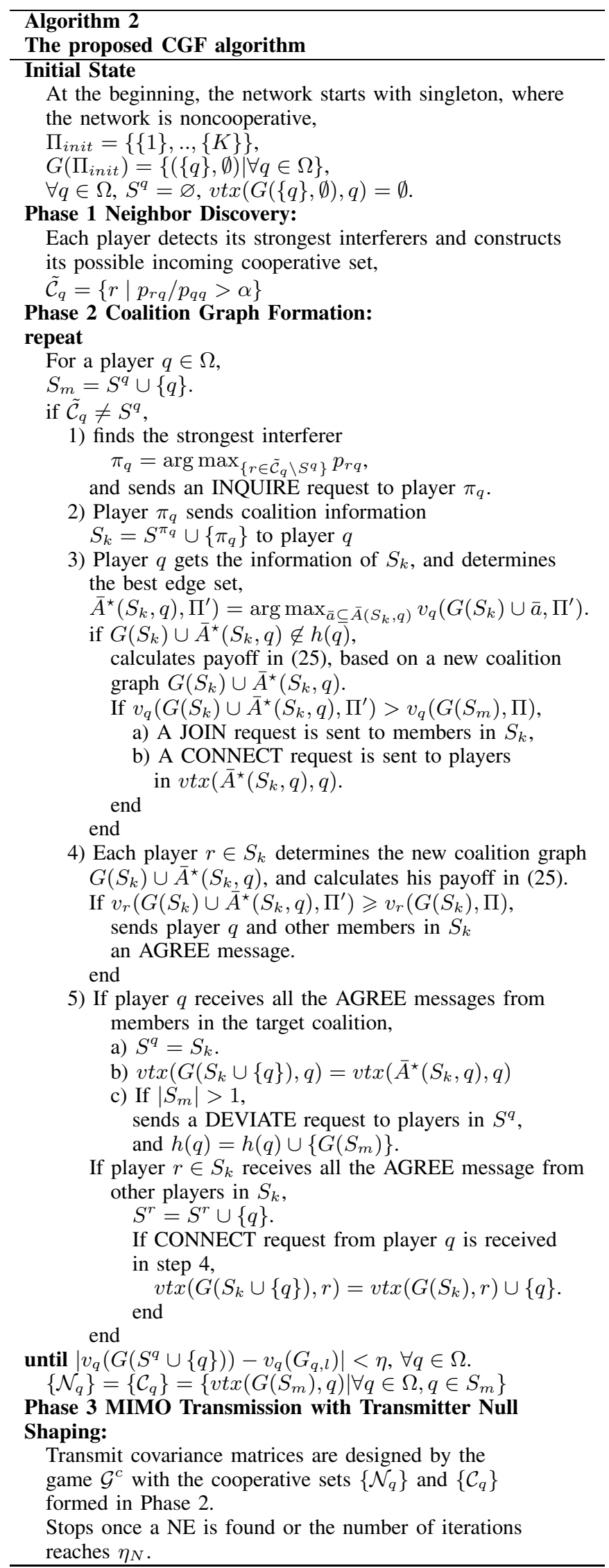

cooperative sets formation problem as a network formation game, and use directed graph to embody the cooperations among players. 
We first introduce some basic concepts of network formation game. A directed graph $G^{D}(S)$ is defined to be a pair $\left(S, A^{D}\right)$, where $S$ is a non-empty finite set of vertices, and $A^{D}$ is a collection of directed arcs of the graph. Let $a_{q r}$ be a directed arc from vertex $q$ to vertex $r$. The set of incoming arcs of vertex $q$ is defined as $A_{q}^{i n}=\left\{a_{r q} \in A^{D} \mid r \in S, q \in\right.$ $S\}$, and the set of outgoing arcs of vertex q is defined as $A_{q}^{\text {out }}=\left\{a_{q r} \in A^{D} \mid r \in S, q \in S\right\}$. Denote $A_{q}^{D}=A_{q}^{\text {in }} \cup A_{q}^{\text {out }}$ as the directed arcs of vertex q. The set of vertices that are origins/destinations of the incoming/outgoing arcs of vertex $\mathrm{q}$ is denoted as $v \operatorname{tx}\left(A_{q}^{i n}\right)$ and $v t x\left(A_{q}^{\text {out }}\right)$ respectively.

The set of possible outgoing arcs from vertex $q$ to other vertices is denoted as $\bar{A}_{q}^{\text {out }}=\left\{a_{q r} \mid r \in S, q \in S\right\}$, while the set of possible incoming arcs from other vertices to vertex $q$ is denoted as $\bar{A}_{q}^{i n}=\left\{a_{r q} \mid r \in S, q \in S\right\}$. The set of all possible directed arcs between vertex $q$ and other vertices is denoted as $\bar{A}_{q}^{D}=\bar{A}_{q}^{\text {in }} \cup \bar{A}_{q}^{\text {out }}$.

Given a directed graph $G^{D}=\left(\Omega, A^{D}\right)$, we denote $v_{q}\left(G^{D}\right)$ as the payoff that player $q \in \Omega$ can receive when graph $G^{D}$ is in place. Consider the transmission rate of each link as the payoff of each player, the value set, i.e., the mapping $V$, can be defined as follows:

$$
V\left(G^{D}\right)=\left\{\mathbf{v}\left(G^{D}\right) \in \mathbb{R}^{|\Omega|}\right\}
$$

where $\forall q \in \Omega, v_{q}\left(A^{D}\right)$ is given by

$$
v_{q}\left(A^{D}\right)=R_{q}^{c}\left(\mathbf{Q}_{q}\left(v t x\left(A_{q}^{\text {out }}\right)\right), \mathbf{Q}_{-v t x\left(A_{q}^{\text {in }}\right)}\right)
$$

Using (28), the cooperative set formation can be modeled as a $\left(\Omega, A^{D}, V\right)$ network formation game. With such a formulation, the outgoing cooperative set $\mathcal{N}_{q}=v t x\left(A_{q}^{\text {out }}\right)$, and the incoming cooperative set $\mathcal{C}_{q}=v t x\left(A_{q}^{i n}\right)$.

In order to present a network formation algorithm, we borrow the concept of potential function from [34]: An exact potential function $\phi$ is a function that maps every strategy vector $\mathbf{s}=\left(s_{1}, s_{2}, . ., s_{M}\right)$ to some real value and satisfies the following conditions: If $s_{q}^{\prime} \neq s_{q}$ is an alternate strategy for player $q$, and $\mathbf{s}^{\prime}=\left(\mathbf{s}_{-q}, s_{q}^{\prime}\right)$, then $\phi(\mathbf{s})-\phi\left(\mathbf{s}^{\prime}\right)=$ $v_{q}(\mathbf{s})-v_{q}\left(\mathbf{s}^{\prime}\right)$.

This definition implies that each player's individual interest is aligned with the group's interest, since each change in the utility function of each player directly represents the same change in the potential function. If players act sequentially, and choose best response strategies or at least improve their utilities (better response strategies), given the most recent actions of the other players, then the game will converge to a $\mathrm{NE}$ regardless of the order of players and the initial condition of the game [34].

Define the potential function $\phi_{q}$ as

$$
\begin{aligned}
\phi_{q}\left(A_{q}^{\prime D}, A_{-q}^{\prime D}\right) & =v_{q}\left(A_{q}^{\prime D}, A_{-q}^{\prime D}\right) \\
& -\sum_{r \neq q}\left(v_{r}\left(A_{q}^{D}, A_{-q}^{D}\right)-v_{r}\left(A_{q}^{\prime D}, A_{-q}^{\prime D}\right)\right) .
\end{aligned}
$$

This expression reflects the intention to maximize the player's own payoff, but subtracting the potential negative effect over other players. Using the potential function in (30), we define the following network switch rule for cooperative sets formation.

Definition 5 (Network Switch Rule): Given a directed arc set $A^{D}$, a player $q$ decides to remove his current $\operatorname{arcs} A_{q}^{D}$ and build new arcs $\bar{A}_{q}^{\prime D}$, leading to a new arc set $A^{\prime D}=$ $A^{D} \backslash A_{q}^{D} \cup \bar{A}_{q}^{\prime D}$, if and only if,

$$
\left\{\begin{array}{l}
v_{q}\left(A_{q}^{\prime D}, A_{-q}^{\prime D}\right)>v_{q}\left(A_{q}^{D}, A_{-q}^{D}\right) \\
\phi_{q}\left(A_{q}^{\prime D}, A_{-q}^{\prime D}\right) \geq v_{q}\left(A_{q}^{D}, A_{-q}^{D}\right) \\
\bar{A}_{q}^{\prime D} \notin h(q), \quad \text { if } A_{q}^{\prime D} \neq \varnothing
\end{array}\right.
$$

The first condition in (31) indicates that the payoff of player $q$ can be strictly improved after removing current arcs and building arcs, and the second one guarantees the sum payoff of all players in the new directed graph will also increase. The third constraint is used to avoid repetitive switches. The three conditions in (31) is also called preference relation, and can be denoted as $\left(A^{\prime D}\right) \succ_{q}\left(A^{D}\right)$ for simplicity.

Now, we propose a network formation (NF) algorithm composed of three phases: neighbor discovery, network formation with potential function, and MIMO transmission with transmitter null shaping.

In Phase 1, each player discovers its strongest interferers. For player $q$, if $p_{r q} / p_{q q}>\alpha$, he will add this link in his possible incoming cooperative set $\tilde{\mathcal{C}}_{q}$.

In Phase 2, players investigate the possibility of network switch. A procedure of network formation is described as follow.

Player $q$ determines the best incoming arc set from the possible incoming cooperative set $\tilde{\mathcal{C}}_{q}$,

$$
\bar{A}_{q}^{i n, \star}=\arg \max _{\bar{a} \subseteq \tilde{\mathcal{C}}_{q}} v_{q}\left(A^{D} \backslash A_{q}^{i n} \cup \bar{a}\right) .
$$

To calculate the new payoff, player $q$ should be able to estimate the interference-plus-noise covariance matrix $\mathbf{R}_{-v t \underline{a}(\bar{a}, q)}\left(\mathbf{Q}_{-v t x(\bar{a}, q)}\right)$.

If $\bar{A}_{q}^{i n, \star} \neq A_{q}^{i n}$ and $\bar{A}_{q}^{i n, \star} \notin h(q)$,

1. An INQUIRE request is sent to players in set $v t x\left(\bar{A}_{q}^{i n, \star}\right)$.

2. After receiving the INQUIRE request, player $r \in$ $v \operatorname{tx}\left(\bar{A}_{q}^{i n, \star}\right)$ acquires the channel matrices $\mathbf{H}_{r q}$, and generates the new $\mathbf{U}_{r}^{\perp}$ assuming that $A_{r}^{\text {out }}=A_{r}^{\text {out }} \cup\left\{a_{r q}\right\}$. The difference of his payoff

$$
\triangle v_{r}=v_{r}\left(A_{q}^{D} \backslash A_{q}^{i n} \cup \bar{A}_{q}^{i n, \star}\right)-v_{r}\left(A^{D}\right),
$$

is estimated and sent back to player $q$.

3. Player $q$ estimates his potential function,

$$
\phi_{q}\left(A_{q}^{D} \backslash A_{q}^{i n} \cup \bar{A}_{q}^{i n, \star}\right)=v_{q}\left(A_{q}^{D} \backslash A_{q}^{i n} \cup \bar{A}_{q}^{i n, \star}\right)+\sum_{r \in v t x\left(\bar{A}_{q}^{i n, \star}\right)} \triangle v_{r}
$$

If $\phi_{q}\left(A_{q}^{D} \backslash A_{q}^{i n} \cup \bar{A}_{q}^{i n, \star}\right)>v_{q}\left(A^{D}\right)$, player $q$ will update his incoming adjacent vertex set $v \operatorname{tx}\left(A_{q}^{i n}\right)=v \operatorname{tx}\left(\bar{A}_{q}^{i n, \star}\right)$, and history set $h(q)=h(q) \cup A^{D}$. A CONFIRMED message is sent to players in set $v t x\left(\bar{A}_{q}^{i n, \star}\right)$.

4. For a player $r \in v t x\left(\bar{A}_{q}^{i n, \star}\right)$, If the CONFIRMED message is received, he will update his outgoing adjacent vertex set, $v t x\left(A_{r}^{\text {out }}\right)=v t x\left(A_{\underline{r}}^{\text {out }}\right) \cup\{q\}$. Hence, a new directed graph $\left(\Omega, A_{q}^{D} \backslash A_{q}^{i n} \cup \bar{A}_{q}^{i n, \star}\right)$ is generated. Denote $A_{q}^{D, l}$ as the last history of directed arc set of player $q$ put into set $h(q)$, Phase 2 ends until $\left|v_{q}\left(A_{q}^{D}\right)-v_{q}\left(A_{q}^{D, l}\right)\right|<\eta$, $\forall q \in \Omega$.

In Phase 3, players transmit data cooperatively in MIMO interference channel, imposing null shaping constraints formed in Phase 2 on the transmitters. There may be multiple NEs. 


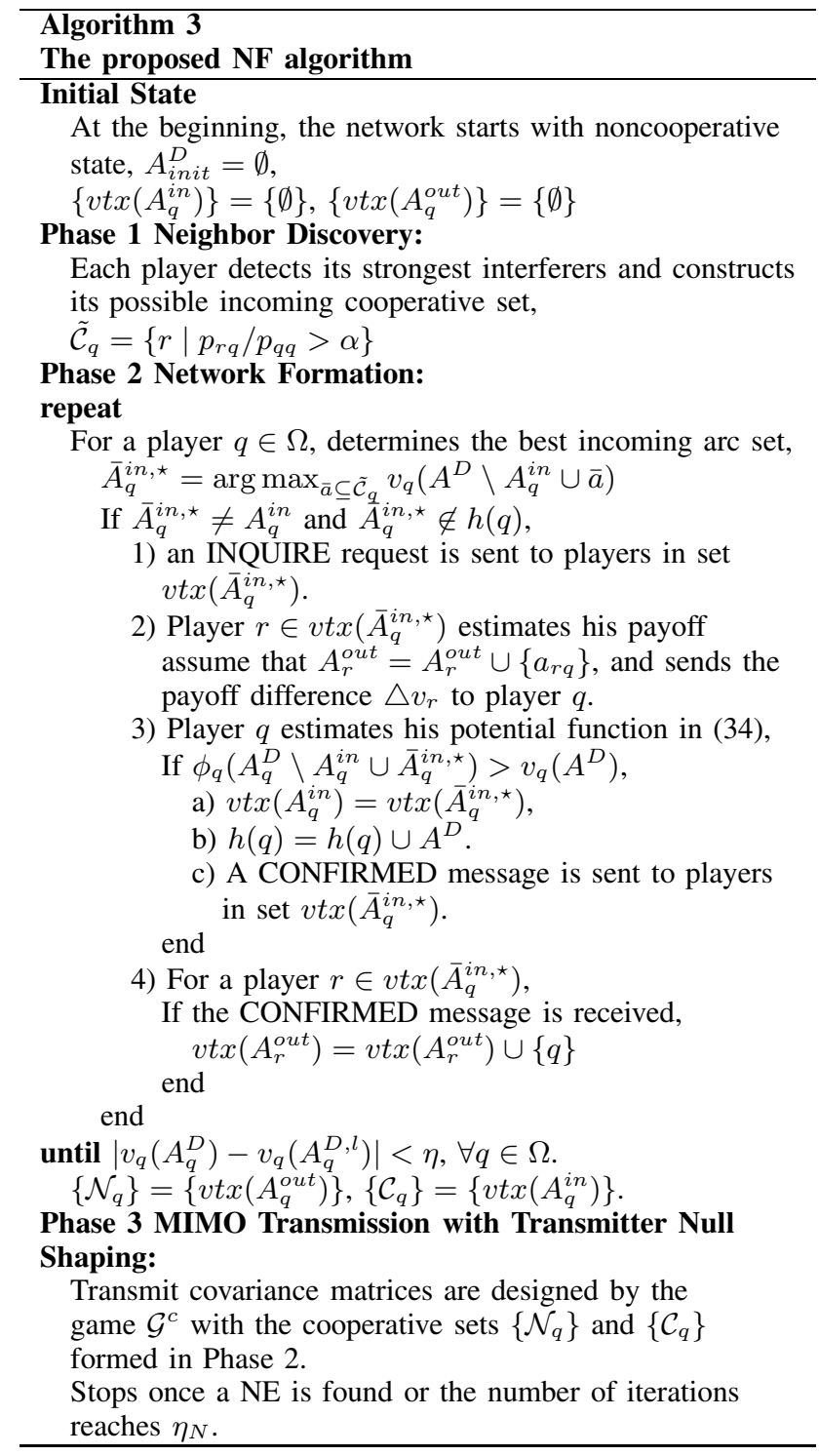

In such cases, we do not search all the NEs and find the best one. Instead, we stop Phase 3 once a NE is found for simplicity, due to which the achieved NE may depend on the initial conditions of the algorithm. If no NE is found within $\eta_{N}$ iterations, Phase 3 stops and each player chooses the strategy in the last iteration.

Theorem 6: The proposed network formation algorithm is stable and always converges to a pure Nash Equilibrium with any initial graph and operation order.

Proof: According to the conclusion of potential game in [29, Th 19.11-19.12], the proposed network formation algorithm converges to a pure NE, since the sum rate of all players is improved in every step. This NE is a directed graph which represents the cooperative sets of each player. The algorithm is stable, due to the monotone convergence property.

\section{Simulation Results And Analysis}

For simulation, we consider a MIMO mutil-cell cellular network, consisting seven hexagonal cells, with full frequency reuse. In each cell, there is one user equipment (UE) being

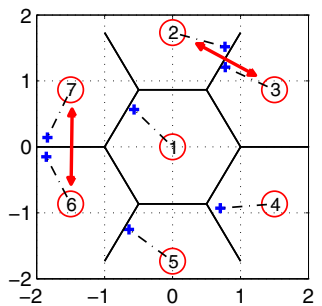

(a) $\mathrm{CF}$

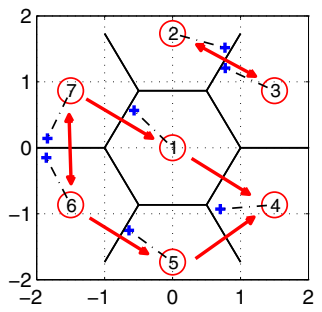

(c) GCG

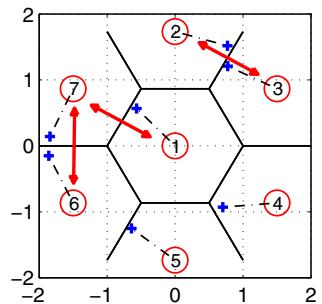

(b) CGF

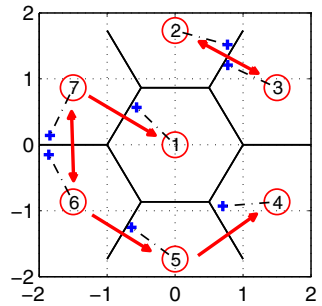

(d) $\mathrm{NF}$
Fig. 2. Snapshot of cooperative sets formation resulting from the proposed $\mathrm{CF}, \mathrm{CGF}$ and NF algorithms with $\left(n_{T_{q}}, n_{R_{q}}\right)=(6,2), n_{l_{q}}=2, d=0.8$ and $P / \sigma^{2}=10 \mathrm{~dB}$.

served by the base station (BS) on a given transmission resource unit. Each UE is randomly distributed with the normalized distance $d \in[0.2,1)$ to its serving BS. The distance between two adjacent BS is $1 \mathrm{~km}$. The elements of the channel matrix $\mathbf{H}_{r q}$ are generated as circularly symmetric complex Gaussian variable. $\mathbf{H}_{r q} \sim \mathcal{C N}\left(0, \eta_{r q}^{2} \mathbf{I}\right)$, with $\eta_{r q}=\sqrt{1 / d_{r q}^{\alpha}}$, and the path loss exponent $\alpha=3$.

In this section, we evaluate the performance of different algorithms through simulations. Specifically, we will evaluate the uniqueness of the NE of noncooperative rate maximization game $\mathcal{G}$ and cooperative transmission game $\mathcal{G}^{c}$. Then we will discuss the influence of the number of transmit antennas and the strength of cross-link interference on different algorithms, and show the effect on average size of outgoing cooperative set $\left|\mathcal{N}_{q}\right|$, computational complexity and average rate. Finally, we will illustrate the influence of SNR levels and different order on the transmission rate.

In Fig. 2, we randomly deploy the users with the normalized distance $d=0.8$, the number of transmit/receiver antennas of each link is $\left(n_{T_{q}}, n_{R_{q}}\right)=(6,2)$, the expected number of data streams $n_{l_{q}}=2$, and $P / \sigma^{2}=10 \mathrm{~dB}$. Fig. 2 shows the features of the proposed $\mathrm{CF}, \mathrm{CGF}$ and NF algorithms. The cooperative sets generated from the proposed coalition algorithms are equivalent to undirected graphs, while the result achieved from the proposed NF algorithm is a directed graph. The solution space of CF algorithm is a subset of that of CGF algorithm. Grand cooperative graph (GCG) represents the altruistic cooperation, where each player helps as many links as possible once condition (8) is satisfied, i.e., $\left|\mathcal{N}_{q}\right|=\left(n_{T_{q}}-n_{l_{q}}\right) / n_{R_{r}}$. In the case of $\left(n_{T_{q}}, n_{R_{q}}\right)=(6,2)$ and $n_{l_{q}}=2,\left|\mathcal{N}_{q}\right|=2$.

Table 1 displays the transmission rates of each players using different algorithms. The proposed CF algorithm achieves higher average rate than that of noncooperative IWF algorithm described in section II, since interference mitigation are performed in coalitions. For example, the transmission rates 
TABLE I

LINK RATE TABLE

\begin{tabular}{|c|c|c|c|c|c|}
\hline Algorithm & NF & GCG & CGF & CF & IWF \\
\hline Link1 & 4.3495 & 3.8666 & 3.8625 & 3.3508 & 3.5538 \\
\hline Link2 & 3.7873 & 4.0089 & 4.1751 & 3.6261 & 3.3242 \\
\hline Link3 & 3.3529 & 3.2130 & 3.4927 & 3.4678 & 1.6417 \\
\hline Link4 & 4.7973 & 5.8439 & 3.5411 & 3.6356 & 3.7098 \\
\hline Link5 & 3.5474 & 2.9140 & 2.6905 & 2.7813 & 2.8444 \\
\hline Link6 & 2.7160 & 2.8621 & 3.5497 & 3.5446 & 2.9776 \\
\hline Link7 & 4.8720 & 4.3866 & 5.5889 & 5.6512 & 4.3218 \\
\hline Rate Per Link & 3.9175 & 3.8707 & 3.8429 & 3.7225 & 3.1962 \\
\hline
\end{tabular}

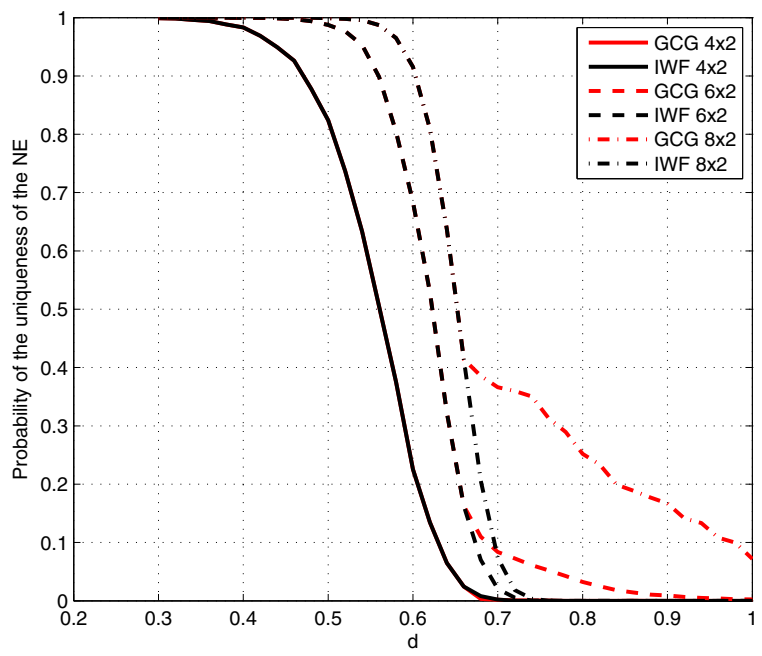

Fig. 3. Probability of uniqueness of the NE of $\mathcal{G}$ and $\mathcal{G}^{c}$ with grand cooperative graph, $\left(n_{T_{q}}, n_{R_{q}}\right)=\{(4,2),(6,2),(8,2)\}, n_{l_{q}}=2$, and $P / \sigma^{2}=5 \mathrm{~dB}$.

of players in coalitions $\{2,3\}$ and $\{6,7\}$ are higher than the rates when they act noncooperative. The proposed CGF further improves $\mathrm{CF}$ by optimizing the cooperative structure in the coalitions. For instance, the coalition graph $\{\{6,7\},\{1,7\}\}$ has higher sum rate than the structure $\{6,7\} \cup\{1\}$ does. The proposed NF algorithm is the best of these algorithms, because it breaks through the limitation of reciprocal cooperation, and expands the optimization dimensions. For example, player 1, 4,5 are more suitable to get unreciprocal helps from their neighbors.

In Fig. 3, we compare the probability of the uniqueness of the $\mathrm{NE}$ of noncooperative rate maximization game $\mathcal{G}$ and cooperative transmission game $\mathcal{G}^{c}$. Here, we use the probability of sufficient condition in Theorem 3 being satisfied to indirectly show the probability of the uniqueness of the NE. We simulate different numbers of the transmit/receiver antennas $\left(n_{T_{q}}, n_{R_{q}}\right)=\{(4,2),(6,2),(8,2)\}$, the expected number of data streams $n_{l_{q}}=2$, and $P / \sigma^{2}=5 \mathrm{~dB}$. Here, we use the simplest GCG to represent the cooperative transmission game. As shown in Fig. 3, the probability of uniqueness of the NE of both games $\mathcal{G}$ and $\mathcal{G}^{c}$ with GCG decreases as the distance between UE and BS increases, corresponding to an increase of inter-cell interference. This confirms to the definition of matrix $\mathbf{S}$ in (20) and the sufficient condition for the uniqueness of the NE given in Theorem 3. Fig. 2 also shows that, increasing the antennas at the transmitter side leads to a grow of uniqueness. The game $\mathcal{G}^{c}$ with GCG has higher

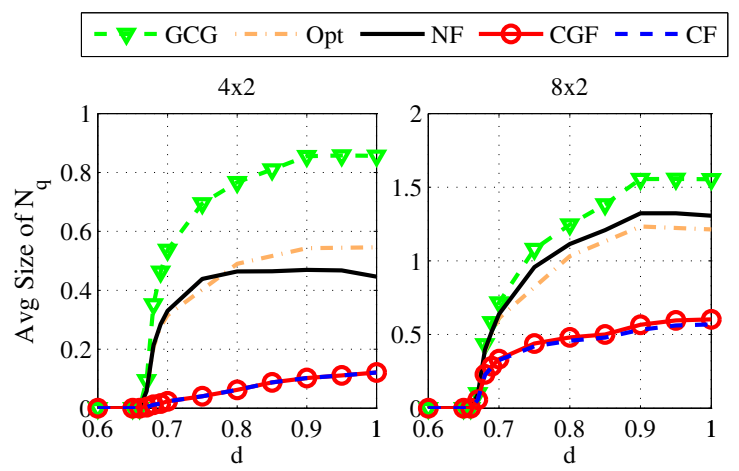

Fig. 4. Average size of set $\mathcal{N}_{q}$ of CF, CGF, NF, GCG and Opt algorithms with regard to $d$ (inter-cell interference). $\left(n_{T_{q}}, n_{R_{q}}\right)=\{(8,2),(4,2)\}, n_{l_{q}}=2$, and $P / \sigma^{2}=5 \mathrm{~dB}$.

TABLE II

\begin{tabular}{|c|c|c|c|c|}
\hline Algorithm & CF & CGF & NF & Opt \\
\hline Payoff-Re-Calculation/Player & 7.9 & 13 & $5 \times 10^{2}$ & $2 \times 10^{4}$ \\
\hline Convergence Steps/Player & 4.6 & 4.8 & 12.6 & $2 \times 10^{4}$ \\
\hline Convergence Time & $2.6 \mathrm{~s}$ & $3.4 \mathrm{~s}$ & $7.1 \mathrm{~s}$ & $46.7 \mathrm{~min}$ \\
\hline
\end{tabular}

probability of uniqueness of $\mathrm{NE}$, compared to the game $\mathcal{G}$, since the power of interference channels are weakened due to cross-link cooperation. The probability difference grows with the number of antennas at the transmitter, since more spatial degrees of freedom are available for interference mitigation.

In Fig. 4-6, we simulate the influence of strength of intercell interference to the proposed CF, CGF, NF algorithms and optimization approach (Opt). Here, the Opt algorithm is based on the proposed NF algorithm with exhaustive search. The results are averaged over random positions of UEs with the normalized distance $d$ varying form 0.6 to 1 , and random realization of the channel matrix. $P / \sigma^{2}=5 \mathrm{~dB}$.

The size of $\mathcal{N}_{q}$ of different algorithms are compared in Fig.4. The parameter $\left|\mathcal{N}_{q}\right|$ represents the number of links a player helps in the MIMO interference systems. From Fig.4, we can see that that all algorithms start the cooperation when $d=0.66$. For $d<0.66$, a user may not suffer strong interference from its neighboring BS. $\left|\mathcal{N}_{q}\right|$ of GCG scheme is largest, since it is the most altruistic scheme of all the algorithms. $\left|\mathcal{N}_{q}\right|$ of Opt is slightly smaller than that of the NF when $n_{T_{q}}=8$, and $n_{T_{q}}=4$ with $d<0.77$, and larger than that of the NF when $n_{T_{q}}=4$ with $d>0.77$. In the $\left(n_{T_{q}}, n_{R_{q}}\right)=(8,2)$ case, when $d>0.77,\left|\mathcal{N}_{q}\right|$ of GCG is larger than 1 , which means that the average number of links requiring interference cancellation is larger than the maximum $\left|\mathcal{N}_{q}\right|$ of the $\left(n_{T_{q}}, n_{R_{q}}\right)=(4,2)$ case. This implies that, compared to the NF algorithm, the Opt approach leads to a smaller $\left|\mathcal{N}_{q}\right|$ when the strong interference leakage can be fully cancelled by the transmitter, and a larger $\left|\mathcal{N}_{q}\right|$ when the transmitter cannot handle all the significant interference leakage to other links. The proposed CF and CGF algorithms have almost the same $\left|\mathcal{N}_{q}\right|$. The $\left|\mathcal{N}_{q}\right|$ of CF/CGF is much smaller than other cooperative algorithms, since the requirement of reciprocality restricts the cooperation among links. $\left|\mathcal{N}_{q}\right|$ of CGF is slightly larger than that of CF when $\left(n_{T_{q}}, n_{R_{q}}\right)=(8,2)$, due to the flexibility introduced in the coalition. 


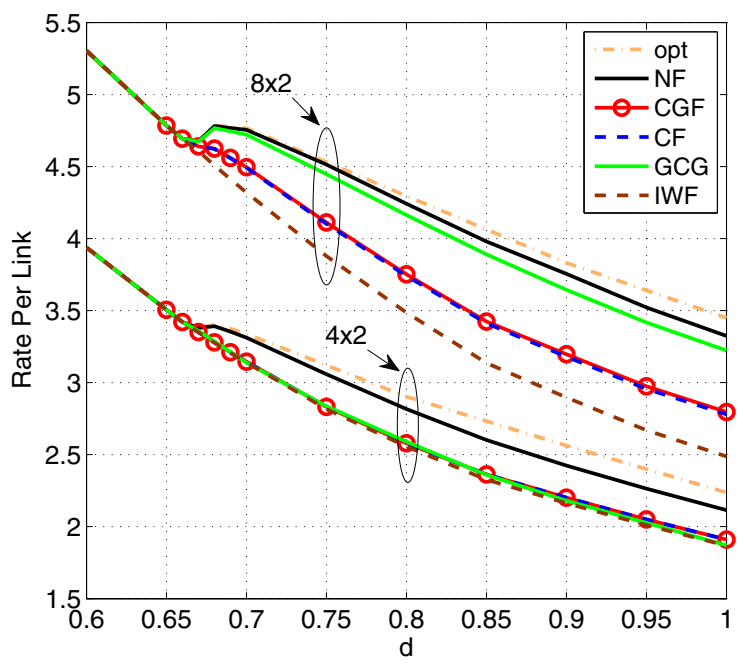

Fig. 5. Average rate of IWF, GCG, CF, CGF, NF and Opt algorithms with regard to $d$ (inter-cell interference). $\left(n_{T_{q}}, n_{R_{q}}\right)=\{(8,2),(4,2)\}, n_{l_{q}}=2$, and $P / \sigma^{2}=5 \mathrm{~dB}$.

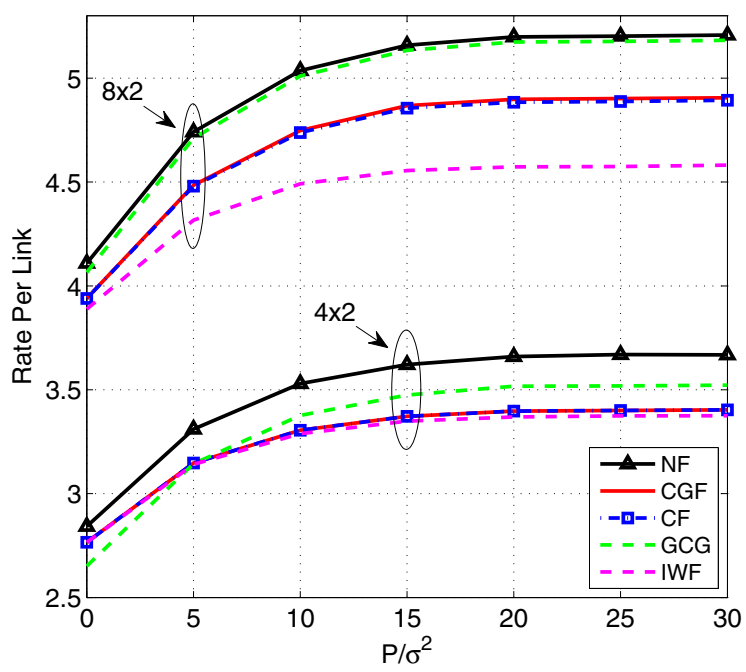

Fig. 6. Average rate of the IWF and proposed CF, CGF and NF algorithms with regard to different $P / \sigma^{2}$. $\left(n_{T_{q}}, n_{R_{q}}\right)=\{(4,2),(8,2)\}, n_{l_{q}}=2$, and $d=0.7$.

In Table II, we illustrate the computational complexity and convergence time of different algorithms when $n_{T_{q}}=8$, $d=0.9, \eta=10^{-4}$, and the time for message exchange among BSs is $20 \mathrm{~ms}$. Since a player investigates possible cooperation switch by re-calculating his payoff, we use the number of times of Payoff-Re-Calculation per player as a measure of computational complexity. We also measure the number of times that Phase 2 is run and denote it as the number of convergence step. From Table II, we can see that the computational complexity of the Opt algorithm that performs full search is too high to be used in practice. On the other hand, the convergence time of the proposed CF, CGF and $\mathrm{NF}$ algorithms are reasonable and applicable to slow mobile system.

In Fig.5, we compare the average transmission rate of the proposed $\mathrm{CF}, \mathrm{CGF}$ and $\mathrm{NF}$ algorithms with the noncooperative
IWF, GCG and Opt. As seen from Fig.5, the average transmission rate of all algorithms generally decrease as users are away from their serving BSs. There is an apparent rise around $d=0.66$ for some algorithms, since cross-link cooperation begins, which corresponds to the result of Fig.4. According to Table II, the complexity of NF algorithm is much less than the Opt, but the transmission rate of NF is close to that of Opt. For the CF, CGF and GCG algorithms, advantages over the noncooperative IWF are shown only when $n_{T_{q}}=8$.

In the proposed $\mathrm{CF}$ algorithm, we assume that any two players in a coalition help each other, i.e., the cross-link interference between any two players in a coalition is mitigated. The outgoing/incoming cooperative set of a player can be represented by the coalition that the player belongs to directly. In this subsection, we will formulate the cooperative sets formation problem as a coalition graph formation game and propose a coalition graph formation (CGF) algorithm. In the proposed CGF algorithm, wether the cross-link interference between two players in a coalition is canceled or not depend on the structure of the coalition graph.

In Fig. 6, we show the average rate achieved per link with regard to different SNR $\left(P / \sigma^{2}\right)$ levels. The results are averaged over random positions of UEs with normalized distance $d=0.7$ and random realization of the channel matrix. The performance of the proposed CF, CGF and NF algorithms are compared with the classic noncooperative IWF algorithm. As seen from Fig. 3, the average rates of all the proposed algorithms and noncooperative IWF algorithm increase as $P / \sigma^{2}$ grows and finally reach their upper bounds. Increasing the antennas at the transmitter leads to higher average rate for all algorithms. When the number of antennas at the transmitter is 8 , the proposed CF and CGF algorithms significantly improve the noncooperative IWF algorithm, and the simple GCG scheme achieves almost the same rate as the proposed NF algorithm. When the number of antennas at the transmitter is 4, CF and CGF algorithms do not show obvious advantages, and GCG scheme even performs worse than IWF at low SNR region. Fortunately, the proposed NF algorithm is apparently superior to the noncooperative IWF with the different SNR and numbers of antennas at the transmit side.

In Fig. 7, we evaluate the influence of different order on the proposed algorithms. The users are uniformly located with the normalized distance $d=0.7$ to their serving BS, the number of antennas is set to $\left(n_{T_{q}}, n_{R_{q}}\right)=(8,2)$, and the expected number of data streams $n_{l_{q}}=2$. The cross ' + ' represents the variance of transmission rate over random positions of UE and random realization of the channel matrix, while the circle 'o' stands for the variance of transmission rate over random positions of UE, random realization of the channel matrix, and random generation of the operation order. Here, for clearness, we only display the variances of CGF and NF, since CF and CGF exhibit quite similar characteristics. As seen from Fig.4, the centers of each $\{+, \circ\}$ pair largely coincide, which indicates that different order has negligible effect on the property of the proposed algorithms.

Considering the performance evaluated in this section, we summarize the properties of the proposed algorithms in Table III. 
TABLE III

ALGORITHM COMPARISON

\begin{tabular}{|c|c|c|c|c|}
\hline Algorithm & Brief Concept & Advantage & Disadvantage & Application \\
\hline IWF & Noncooperative & Lowest complexity, rapid convergence & Low rate & Slow mobility \\
\hline $\mathrm{CF}$ & $\begin{array}{l}\text { Reciprocal cooperation, } \\
\text { Cooperative sets of a player is revealed } \\
\text { by all members in the coalition without } \\
\text { any structure among members. }\end{array}$ & $\begin{array}{l}\text { Low complexity, rapid convergence, } \\
\text { improved rate when } n_{T_{q}} \text { is large }\end{array}$ & $\begin{array}{l}\text { Low rate } \\
\text { when } n_{T_{q}} \text { is small }\end{array}$ & $\begin{array}{l}\text { Slow mobility, } \\
\text { large } n_{T_{q}}\end{array}$ \\
\hline CGF & $\begin{array}{l}\text { Reciprocal cooperation, } \\
\text { Cooperative sets of a player is revealed } \\
\text { by the graph structure of members in } \\
\text { the coalition }\end{array}$ & $\begin{array}{l}\text { Low complexity, rapid convergence, } \\
\text { improved rate when } n_{T_{q}} \text { is large }\end{array}$ & $\begin{array}{l}\text { Low rate } \\
\text { when } n_{T_{q}} \text { is small }\end{array}$ & $\begin{array}{l}\text { Slow mobility, } \\
\text { large } n_{T_{q}}\end{array}$ \\
\hline GCG & Altruistic nonreciprocal cooperation & $\begin{array}{l}\text { Lowest complexity, rapid convergence, } \\
\text { approaches the rate of NF at high SNR }\end{array}$ & $\begin{array}{l}\text { Deteriorative rate } \\
\text { at low SNR }\end{array}$ & $\begin{array}{l}\text { Slow mobility, } \\
\text { high SNR }\end{array}$ \\
\hline $\mathrm{NF}$ & $\begin{array}{l}\text { Nonreciprocal cooperation based on } \\
\text { potential function }\end{array}$ & $\begin{array}{l}\text { Rapid convergence, } \\
\text { approaches the rate of Opt }\end{array}$ & High complexity & Slow mobility \\
\hline Opt & $\begin{array}{l}\text { Nonreciprocal cooperation based on } \\
\text { potential function with exhaustive } \\
\text { search }\end{array}$ & Highest achievable rate & $\begin{array}{l}\text { Very high complexity, } \\
\text { slow convergence }\end{array}$ & Static scenario \\
\hline
\end{tabular}

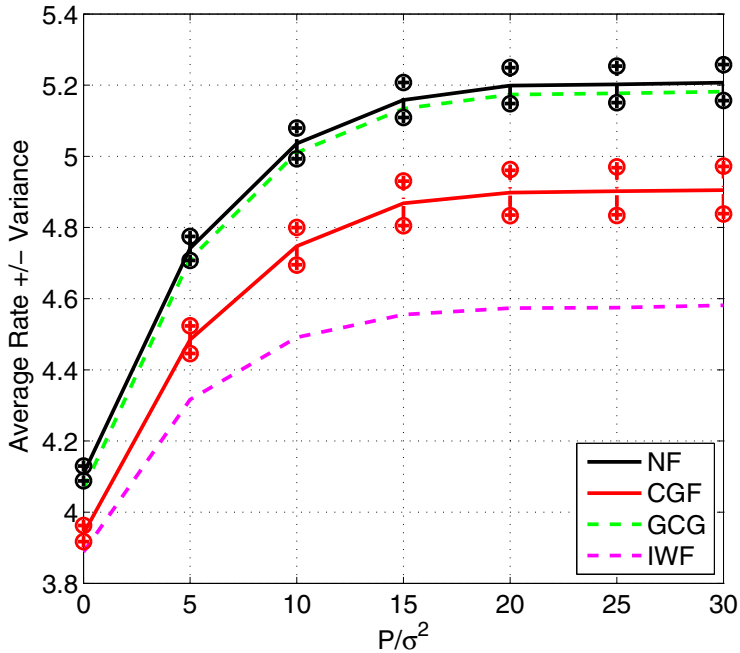

Fig. 7. Influence of difference order on the proposed CGF and NF algorithms, $\left(n_{T_{q}}, n_{R_{q}}\right)=(8,2), n_{l_{q}}=2$, and $d=0.7$.

\section{CONCLUSION}

In this paper, we consider the cooperative maximization of mutual information in the MIMO Gaussian interference channel via game theory. Null shaping constraints are enforced in the design of transmit covariance matrices to enable interference mitigation among links. The transmit covariance matrices leading to the $\mathrm{NE}$ are derived, and the existence and uniqueness of the NE is analyzed. We define cooperative sets to stand for the cooperation relationship among links, and the formation of the cooperative sets is formulated as coalition games and network formation games. The proposed CF and CGF algorithms are Nash-stable, and the proposed NF algorithm converges to a Nash Equilibrium. Simulation results show that CF and CGF algorithms show significant advantages when the antennas at the transmitters is large, and the NF algorithm enhances the sum rate of the system apparently even at low SNR region or with small number of transmit antennas.

\section{REFERENCES}

[1] T. M. Cover and J. A. Thomas, Elements of Information Theory. Wiley, 1991.

[2] T. Kwon, H. Lee, S. Choi, J. Kim, D. H. Cho, S. Cho, S. Yun, W. H. Park, K. Kim, "Design and implementation of a simulator based on a cross-layer protocol between MAC and PHY layers in a WiBRO compatible IEEE 802.16e OFDMA system," IEEE Commun. Mag., vol. 43, no. 12, pp. 136-146, Dec. 2005.

[3] X. Wang and R. Gharpurey, "Interference cancellation in broadband wireless systems utilizing phase-aligned injection-locked oscillators," IEEE Trans. Circuits Syst. II: Exp. Briefs, vol. 55, no. 9, pp. 872-876, Sept. 2008.

[4] F. Gomez-Cuba, R. Asorey-Cacheda, F. Gonzalez-Castano, "A survey on cooperative diversity for wireless networks," IEEE Commun. Surveys \& Tuts., vol. 13, no. 99, pp. 1-14, 3rd Quarter 2011.

[5] T. Zhou, M. Peng, W. Wang, and H. Chen, "Low-complexity coordinated beamforming for downlink multi-cell SDMA/OFDM system," IEEE Trans. Veh. Technol. vol. 62, no. 1, pp. 247-255, Jan. 2013.

[6] S. W. Halpern, "Reuse partitioning in cellular systems," in Proc. 1983 IEEE Veh. Technol. Conf. - Spring, vol. 33, pp. 322-327.

[7] 3GPP R1-050507, Huawei, "Soft frequency reuse scheme for UTRAN LTE," TSG RAN WG1 Meeting \#41, Athens, Greece, May 2005.

[8] S. G. Kiani, D. Gesbert, A. Gjendemsjo, and G. Oien, "Distributed power allocation for interfering wireless links based on channel information partitioning," IEEE Trans. Wireless Commun., vol. 8, no. 6, pp. 3004-3015, June 2009.

[9] C. Liang and K. R. Dandekar, "Power management in MIMO ad hoc networks: a game-theoric approach," IEEE Trans. Wireless Commun., vol. 6, no. 4, pp. 1164-1170, Apr. 2007.

[10] G. Scutari, D. P. Palomar, and S. Barbarossa, "The MIMO iterative waterfilling algorithm," IEEE Trans. Signal Process., vol. 57, no. 5, pp. 1917-1935, May 2009.

[11] G. Scutari, D. P. Palomar, and S. Barbarossa, "Competitive design of multiuser MIMO systems based on game theory: a unified view," IEEE J. Sel. Areas Commun., vol. 26, no. 7, pp. 1089-1103, Sept. 2008.

[12] B. Wang, Y. Wu, and K. J. R. Liu, "Game theory for cognitive radio networks: an overview," Comput. Netw., vol. 54, no. 14, pp. 2537-2561, Oct. 2010.

[13] C. Shi, D. A. Schmidt, R. A. Berry, M. L. Honig, and W. Utschick, "Distributed interference pricing for the MIMO interference channel," in Proc. 2009 IEEE ICC, pp. 1796-1800.

[14] J. Zhang, Y. Liu, G. Xie, P. Deng, J. Mao, and H. F. Rashvand, "Taylor approximation pricing for K-user multiple-input multiple-output (MIMO) interference channels," IET Commun., vol. 6, no. 17, pp. $2957-$ 2967, 2012.

[15] Z. Chen, S. A. Vorobyov, C.-X. Wang, and J. S. Thompson, "Nash bargaining over MIMO interference systems," in Proc. 2009 IEEE ICC.

[16] K. M. Ho and D. Gesbert, "Balancing egoism and altruism on the interference channel: The mimo case," in Proc. 2010 IEEE ICC.

[17] V. L. Prasad and N. X. D. Wang, "Coordinated scheduling and power allocation in downlink multicell OFDMA networks," IEEE Trans. Veh. Technol., vol. 58, no. 6, pp. 2835-2848, July 2009. 
[18] S. G. Kiani and D. Gesbert, "Optimal and distributed scheduling for multicell capacity maximization," IEEE Trans. Wireless Commun., vol. 7, no. 1, pp. 288-297, Jan. 2008.

[19] W. Choi and J. Andrews, "The capacity gain from intercell scheduling in multianetnna systems," IEEE Trans. Wireless Commun., vol. 7, no. 2, pp. 714-725, Feb. 2008.

[20] V. R. Cadambe and S. A. Jafar, "Interference alignment and degrees of freedom of the K-user interference channel," IEEE Trans. Inf. Theory, vol. 54, pp. 3425-3441, Aug. 2008.

[21] K. Gomadam, V. R. Cadambe, and S. A. Jafar, "Approaching the capacity of wireless networks through distributed interference alignment," in Proc. 2008 Globecom.

[22] B. N.-Makouei, J. G. Andrews, and R. W. Heath, Jr., "MIMO interference alignment over correlated channels with imperfect CSI," IEEE Trans. Signal Process., vol. 59, no. 6, pp. 2783-2794, June 2011.

[23] E. A. Jorswieck and E. G. Larsson, "Complete characterization of Pareto boundary for the MISO interference channel," IEEE Trans. Signal Process., vol. 56, no. 10, pp. 5292-5296, Oct. 2008.

[24] J. Park and Y. Sung "On the Pareto-optimal beam structure and design for multi-user MIMO interference channels." Available: http://arxiv.org/ abs/1211.4213.

[25] G. Scutari and D. P. Palomar, "MIMO cognitive radio: a game theoretical approach," IEEE Trans. Signal Process., vol. 58, no. 2, pp. 761-780, Feb. 2010.

[26] W. Saad, Z. Han, M. Debbah, A. Hjrungnes, and T. Basar, "Coalitional game theory for communication networks," IEEE Signal Process. Mag., vol. 26, no. 5, pp. 77-97, Sept. 2009.

[27] W. Saad, Z. Han, R. Zheng, et al., "Coalitional games in partition form for joint spectrum sensing and access in cognitive radio networks," IEEE Trans. Sel. Topics Signal Process., vol. 6, no. 2, pp. 195-209, Apr. 2012.

[28] Z. Han, Z. Ji, and K. J. R. Liu, "Fair multiuser channel allocation for OFDMA networks using Nash bargaining solutions and coalitions," IEEE Trans. Commun., vol 53, no 8, pp. 1366-1376, Aug. 2005.

[29] N. Nisan, T. Roughgarden, E. Tardos, and V. Vazirani, Algorithmic Game Theory. Cambridge University Press, 2007.

[30] D. Fudenberg and J. Tirole, Game Theory. MIT Press, 1991.

[31] R. Mochaourab and E. Jorswieck, "Coalition formation in MISO interference channels," in Proc. 2011 IEEE International Workshop Computational Advances Multi-Sensor Adaptive Process.

[32] Y. Hao and V. K. N. Lau, "Rank-constrained Schur-convex optimization with multiple trace/log-det constraints," IEEE Trans. Signal Process., vol. 59, no. 1, pp. 304-314, Jan. 2011.

[33] A. Bogomonlaia and M. Jackson, "The stability of hedonic coalition strctures," Games Econ. Behav., vol. 38, pp. 201-230, Jan. 2002.

[34] J. R. Gallego, M. Canales, and J. Ortin, "Distributed resource allocation in cognitive radio networks with a game learning approach to improve aggregate system capacity," Elsevier Ad Hoc Netw., vol. 10, no. 6, pp. 1076-1089, 2012.

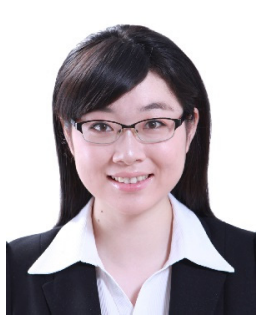

Tong Zhou received the B.S. degree from the School of Information and Communication Engineering, Beijing University of Posts \& Communications (BUPT), China, in 2008. She is currently working toward the Ph.D. degree with the Key Laboratory of Universal Wireless Communications (Ministry of Education), BUPT. From September 2011 to August 2013, she visited the Signals and Information Group (SIG) at Department of Electrical \& Computer Engineering (ECE) of University of Maryland, supported by China Scholarship Council (CSC) for two years. Her current research interests are interference coordination and resource allocation in multi-cell systems.

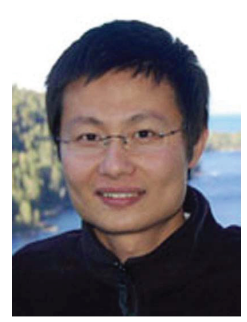

Yan Chen (S'06-M'11) received the Bachelor's degree from University of Science and Technology of China in 2004, the M. Phil degree from Hong Kong University of Science and Technology (HKUST) in 2007, and the Ph.D. degree from University of Maryland College Park in 2011.

$\mathrm{He}$ is currently a research associate in the Department of Electrical and Computer Engineering at University of Maryland College Park. His current research interests are in social learning and networking, smart grid, cloud computing, crowdsourcing, network economics, multimedia signal processing and communication.

Dr. Chen received the University of Maryland Future Faculty Fellowship in 2010, Chinese Government Award for outstanding students abroad in 2011, University of Maryland ECE Distinguished Dissertation Fellowship Honorable Mention in 2011, and was the Finalist of A. James Clark School of Engineering Dean's Doctoral Research Award in 2011.

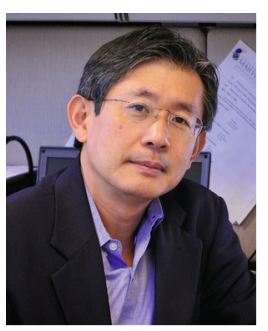

K. J. Ray Liu (F'03) was named a Distinguished Scholar-Teacher of University of Maryland, College Park, in 2007, where he is Christine Kim Eminent Professor of Information Technology. He leads the Maryland Signals and Information Group conducting research encompassing broad areas of signal processing and communications with recent focus on cooperative communications, cognitive networking, social learning and networks, and information forensics and security.

Dr. Liu is the recipient of numerous honors and awards including IEEE Signal Processing Society Technical Achievement Award and Distinguished Lecturer. He also received various teaching and research recognitions from University of Maryland including university-level Invention of the Year Award; and Poole and Kent Senior Faculty Teaching Award and Outstanding Faculty Research Award, both from A. James Clark School of Engineering.

An ISI Highly Cited Author, Dr. Liu is a Fellow of IEEE and AAAS. Dr. Liu is President of IEEE Signal Processing Society where he has served as Vice President Publications and Board of Governor. He was the Editor-inChief of IEEE Signal Processing Magazine and the founding Editor-in-Chief of EURASIP Journal on Advances in Signal Processing. 